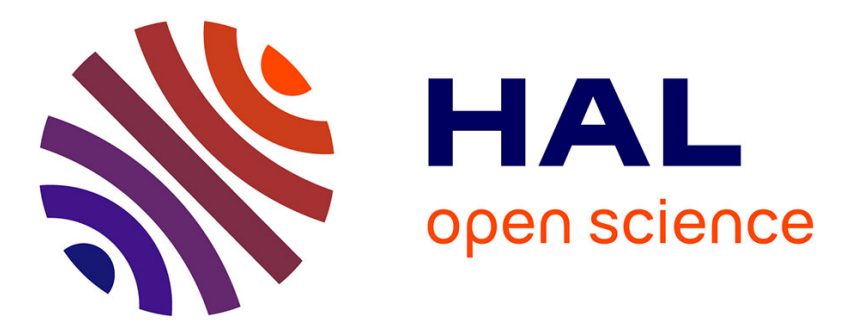

\title{
Scenario development for the observation of alpha-driven instabilities in JET DT plasmas
}

\author{
R. J Dumont, J. Mailloux, V. Aslanyan, M. Baruzzo, C. D. Challis, I. Coffey,
} A. Czarnecka, E. Delabie, J. Eriksson, J. Faustin, et al.

\section{> To cite this version:}

R. J Dumont, J. Mailloux, V. Aslanyan, M. Baruzzo, C. D. Challis, et al.. Scenario development for the observation of alpha-driven instabilities in JET DT plasmas. Nuclear Fusion, 2018, 58, pp.082005. 10.1088/1741-4326/aab1bb . cea-01829932

\section{HAL Id: cea-01829932 https://hal-cea.archives-ouvertes.fr/cea-01829932}

Submitted on 4 Jul 2018

HAL is a multi-disciplinary open access archive for the deposit and dissemination of scientific research documents, whether they are published or not. The documents may come from teaching and research institutions in France or abroad, or from public or private research centers.
L'archive ouverte pluridisciplinaire HAL, est destinée au dépôt et à la diffusion de documents scientifiques de niveau recherche, publiés ou non, émanant des établissements d'enseignement et de recherche français ou étrangers, des laboratoires publics ou privés. 


\title{
Scenario development for the observation of alpha-driven instabilities in JET DT plasmas
}

\author{
R. J. Dumont ${ }^{1}$, J. Mailloux ${ }^{2}$, V. Aslanyan ${ }^{3}$, M. Baruzzo ${ }^{4}$, C. D. \\ Challis $^{2}$, I. Coffey ${ }^{5}$, A. Czarnecka ${ }^{6}$, E. Delabie ${ }^{7}$, J. Eriksson ${ }^{8}$, J. \\ Faustin $^{9}$, J. Ferreira ${ }^{10}$, M. Fitzgerald ${ }^{2}$, J. Garcia ${ }^{1}$, L. \\ Giacomelli $^{11}$, C. Giroud ${ }^{2}$, N. Hawkes ${ }^{2}$, Ph. Jacquet ${ }^{2}$, E. \\ Joffrin $^{1}$, T. Johnson ${ }^{12}$, D. Keeling ${ }^{2}$, D. King ${ }^{2}$, V. Kiptily ${ }^{2}$, B. \\ Lomanowski $^{13}$, E. Lerche ${ }^{14}$, M. Mantsinen ${ }^{15,16}$, L. Meneses ${ }^{10}$, S. \\ Menmuir $^{2}$, K. McClements ${ }^{2}$, S. Moradi ${ }^{2}$, F. Nabais ${ }^{10}$, M. \\ Nocente $^{11}$, A. Patel ${ }^{2}$, H. Patten ${ }^{9}$, P. Puglia ${ }^{9}$, R. Scannell ${ }^{2}$, S. \\ Sharapov $^{2}$, E. R. Solano ${ }^{17}$, M. Tsalas ${ }^{18,19}$, P. Vallejos ${ }^{12}$, H. \\ Weisen $^{9}$ and JET contributorsł. \\ ${ }^{1}$ CEA, IRFM, F-13108 Saint-Paul-lez-Durance, France. ${ }^{2}$ CCFE, Culham Science \\ Centre, Abingdon, OX14 3DB, UK. ${ }^{3}$ MIT PSFC, 175 Albany Street, Cambridge, MA \\ 02039, USA. ${ }^{4}$ Consorzio RFX, corso Stati Uniti 4, 35127 Padova, Italy. ${ }^{5}$ Dept of \\ Pure and Applied Physics, Queens University, Belfast, BT7 1NN, UK. ${ }^{6}$ Institute of \\ Plasma Physics and Laser Microfusion, 00-908 Warsaw, Hery Street 23, Poland. \\ ${ }^{7}$ Oak Ridge National Laboratory, Oak Ridge, Tennessee, US. ${ }^{8}$ Dept of Physics and \\ Astronomy, Uppsala Uni., SE-75119 Uppsala, Sweden. ${ }^{9}$ École Polytechnique Fédérale \\ de Lausanne (EPFL), Swiss Plasma Center (SPC), CH-1015 Lausanne, Switzerland. \\ ${ }^{10}$ Instituto de Plasmas e Fusão Nuclear, IST, Universidade de Lisboa, Portugal. \\ ${ }^{11}$ Uni. Milano-Bicocca, piazza della Scienza 3, 20126 Milano, Italy. ${ }^{12}$ Fusion Plasma \\ Physics, EES, KTH, SE-10044 Stockholm, Sweden. ${ }^{13}$ Aalto Uni., P.O.Box 14100, \\ FIN-00076 Aalto, Finland. ${ }^{14}$ LPP-ERM/KMS, Ass. EUROFUSION-Belgian State, \\ TEC partner, Brussels, Belgium. ${ }^{15}$ Barcelona Supercomputing Center, Barcelona, \\ Spain. ${ }^{16}$ ICREA, Barcelona, Spain. ${ }^{17}$ Laboratorio Nacional de Fusión, CIEMAT, \\ Madrid, Spain. ${ }^{18}$ FOM Institute DIFFER NL-3430 BE Nieuwegein, The \\ Netherlands. ${ }^{19}$ ITER Organization, Route de Vinon sur Verdon, 13067 St Paul Lez \\ Durance, France. \\ E-mail: remi.dumont@cea.fr
}

\begin{abstract}
In DT plasmas, Toroidal Alfvén Eigenmodes (TAEs) can be made unstable by the alpha particles resulting from fusion reactions, and may induce a significant redistribution of fast ions. Recent experiments have been conducted in JET deuterium plasmas in order to prepare scenarios aimed at observing alpha-driven TAEs in a future JET DT campaign. Discharges at low density, large core temperatures associated with the presence of Internal Transport Barriers and characterised by good energetic ion confinement have been performed. ICRH has been used in the hydrogen minority heating regime to probe the TAE stability. The consequent presence of $\mathrm{MeV}$ ions has resulted in the observation of TAEs in many instances. The impact of several
\end{abstract}

$\ddagger$ See the author list of "X. Litaudon et al 2017 Nucl. Fusion 57 102001" 
Scenario development for the observation of alpha-driven instabilities in JET DT plasmas2

key parameters on TAE stability could therefore be studied experimentally. Modeling taking into account NBI and ICRH fast ions shows good agreement with the measured neutron rates, and has allowed predictions for DT plasmas to be performed.

\section{Introduction}

Toroidal Alfvén Eigenmodes (TAE) are weakly damped eigenmodes (gap modes) belonging to a wide class of electromagnetic instabilities[1]. They are readily destabilised by the free energy source in the distribution function of energetic ions and as a result, TAEs have been commonly observed in magnetic fusion plasmas[2]. In typical experiments, these fast ions are usually generated by auxiliary heating methods, either Neutral Beam Injection (NBI) or Ion Cyclotron Resonance Heating (ICRH) systems. In addition to these energetic populations, future fusion reactors will operate with a deuterium-tritium (DT) mixture as fuel. The resulting fusion reactions create helium ions with a birth energy around $3.5 \mathrm{MeV}$ (alpha particles), i.e. well in excess of the plasma temperature. Like other fast ions, alpha particles can destabilise TAEs.

In burning plasmas, alpha-driven TAEs ( $\alpha$-driven TAEs) could play a crucial role in a non-linear interaction with alphas: in a worst-case scenario, the alpha population build-up results in the destabilisation of $\alpha$-driven TAEs, which themselves displace the alphas to more peripheral regions. This could affect the discharge performance by reducing the core alpha power, and even potentially cause significant losses and subsequent large fluxes on the first wall and plasma facing components. Because of the relative scarcity of DT experiments, $\alpha$-driven TAEs have not been as well studied as ICRH- or NBI-driven TAEs. Given the uncertainties related to the effects of these MHD instabilities in next-step devices including ITER $[3,4,5,6]$, it is highly desirable to document the features of $\alpha$-driven TAEs as extensively as possible both from a theoretical and an experimental standpoint in ongoing fusion experiments.

In the past, significant efforts have been devoted to observe and characterise $\alpha$ driven TAEs in the two only magnetic fusion devices capable so far of producing DT plasmas: the Tokamak Fusion Test Reactor (TFTR) and the Joint European Torus (JET). Despite optimistic initial predictions[7], observing $\alpha$-driven TAEs in TFTR actually proved to be quite challenging, requiring scenario fine-tuning guided by numerical simulation[8]. Eventually, however, this endeavour resulted in the unambiguous detection of $\alpha$-driven TAEs for the first time in a DT experiment $[9,10]$. In JET, on the other hand, it was established that $\alpha$-driven TAEs could not be destabilised in hot ion $\mathrm{H}$ mode plasmas because of the large central bulk plasma pressure. On the other hand, numerous TAEs have been routinely observed in shear optimised discharges featuring Internal Transport Barriers (ITBs). However, the use of ICRH power to create the ITBs, even at relatively low levels of power ( $\gtrsim 1 \mathrm{MW})$, provides a TAE drive in excess of the alpha drive, and there were significant uncertainties in its precise magnitude. As 
a result, no firm conclusions regarding $\alpha$-driven TAEs could be drawn from past DT experiments in JET[11, 12, 13].

We report here on an effort to develop a scenario optimal for the observation of alpha-driven TAEs in a forthcoming DT campaign in JET, guided by past results in JET, TFTR and numerical calculations. In section 2, the parameters adapted to the observation of $\alpha$-driven TAEs in DT discharges are identified. Observations of ITBs and consequences in terms of plasma quantities are reported in section 3. In section 4, the performance obtained in terms of neutron production in these discharges is discussed, and the precise role of ICRH is elucidated. TAE stability of these pulses is examined in section 5 and predictions for DT plasmas is the subject of section 6 .

\section{Operational regime}

The parameters pertaining to the observation of $\alpha$-driven TAEs may be identified by examining the balance between the TAE drive by alphas $\left(\gamma_{\alpha}\right)$ and the damping by different mechanisms $\left(\gamma_{d}\right)$, which can be expressed in the following simplified form $[14,15,16]$

$$
\gamma \equiv \gamma_{\alpha}-\gamma_{d}=-C q^{2} \beta_{T \alpha}\left(1-\frac{\omega_{\alpha}^{\star}}{\omega}\right) F\left(v_{\alpha} / v_{A}\right)-\gamma_{d},
$$

with $q$ the safety factor, $\beta_{T \alpha}$ the alpha normalised pressure, $\omega$ the mode frequency. $\omega_{\alpha}^{\star}$ is the $\alpha$ diamagnetic frequency, $F$ is a function of the normalised $\alpha$ birth velocity $\left(v_{\alpha}\right)$, $v_{A}$ is the Alfvén velocity and $C$ is a constant.

The destabilisation of TAE is only possible when the alpha diamagnetic drift frequency exceeds the eigenfrequency, i.e.

$$
\omega_{\alpha}^{\star} \equiv \frac{m}{r} \frac{T_{\alpha}}{q_{\alpha} B} \frac{d \log \left(p_{\alpha}\right)}{d r}>\omega .
$$

In the latter expression, $m$ is the poloidal mode number, $r$ is the minor radius, $T_{\alpha}$ is the alpha temperature, $q_{\alpha}$ is the electric charge of alpha particles, $B$ is the confinement field and $p_{\alpha}$ is the alpha pressure. This condition defines a threshold in terms of the alpha pressure gradient. Furthermore, Eq. 1 clearly shows that both $\beta_{\alpha}$ and $q$ should be maximised.

As discussed in Ref. [11], the large bulk plasma pressure has prevented TAEs from being driven unstable in typical fusion performance H-mode scenarios performed during the previous JET DT campaign, despite a large alpha drive. On the other hand, TAEs have been observed in shear-optimised discharges as a result of the elevated $q$ profile, but only in the presence of ICRH power since it was essentially employed in all these pulses. The potential presence of radiofrequency-generated energetic ions in the plasma has prevented the $\alpha$-driven TAEs from being unequivocally observed and characterised in JET. In order to alleviate this risk in a future DT campaign, no ICRH will be used before the time of interest for $\alpha$-driven TAE observation, i.e. the peak performance phase of the DT discharge and the afterglow phase. In order to maximise the alpha drive in this context, it is thus necessary to make use of the full capability of the NBI system in 
JET to maximise the fusion reaction yield by increasing $T_{i}$, the ion temperature, since the thermonuclear reaction rate increases rapidly with the fuel temperature. On the other hand, in order to maximise the effect of energetic ions, it is desirable to maximise the fast ion pressure, which is proportional to the Spitzer slowing down time $\tau_{s} \propto T_{e}^{3 / 2}$ with $T_{e}$ the electron temperature[17]. High electron temperatures are best achieved by operating at relatively low densities. In H-mode plasmas fuelled by NBI ions, this translates into operating at low plasma current $I_{p}$, with the constraint that the alpha particle confinement remain satisfactory.

We now turn to the mode damping, which should be sufficiently low to be overcome by the alpha-drive. For gap Alfvén Eigenmodes (AEs), this total damping is a combination of radiative damping $\left(\gamma_{\text {rad }}\right)$, collisional electron damping $\left(\gamma_{\text {colle,e}}\right)$, thermal electron $\left(\gamma_{e}\right)$ and ion $\left(\gamma_{D}, \gamma_{T}\right)$ Landau damping and damping by NBI ions $\left(\gamma_{N B I}\right)$ fulfilling the condition $v_{\|}=v_{A} / 3$. We find that in JET, this last mechanism is largely dominant. Calculations performed with the CASTOR-K and MISHKA codes during the last DT campaign yield $\gamma_{N B I} / \omega \sim 1-2 \%$ whereas the maximum alpha drive was estimated to be $\gamma_{\alpha} / \omega \sim 0.15 \%$, in other words much lower than the damping rate[11]. In TFTR and in JET, the afterglow scheme has therefore been developed to remedy this issue. It consists of switching-off the auxiliary power abruptly, and relying on the more rapid slowing-down of fast NBI deuterons compared to alphas to observe the $\alpha$-driven TAEs in the afterglow phase[9]. The afterglow scheme lends itself to the observation of $\alpha$-driven TAEs if the following condition is satisfied

$$
\left|\gamma_{N B I} e^{-t / \tau_{N B I}}+\left(\gamma_{D}+\gamma_{T}\right) e^{-t / \tau_{E}}+\gamma_{e}\right|<\gamma_{\alpha} e^{-t / \tau_{\alpha}} .
$$

$\tau_{E}$ is the energy confinement time and it is assumed that electron damping remains constant during this phase. $\tau_{N B I}$ (resp. $\tau_{\alpha}$ ) is the typical time required for NBI ions (resp. alphas) to thermalise. Assuming a JET DT plasma with electron density $n_{e}=5 \times 10^{19} \mathrm{~m}^{-3}$ and background temperature $T=10 \mathrm{keV}$, we find that the Spitzer slowing-down time for alphas is $\tau_{s, \alpha} \approx 0.72 \mathrm{~s}$, whereas $\tau_{s, N B I} \approx 1.81 \mathrm{~s}$ for NBI (assuming DT beams) ions. However, due to their larger initial energy, the thermalisation time for alphas is found to be $\tau_{\alpha} \approx 1.20 \mathrm{~s}$, i.e. appreciably longer than the typical time for $100 \mathrm{keV}$ NBI ions to thermalise $\tau_{N B I} \approx 0.31 \mathrm{~s}$. $\tau_{E}$ is estimated to be of the same order as $\tau_{N B I}$ in these discharges. This is consistent with more sophisticated analyses in TFTR and JET which find that the alpha drive remains significant after the beam and thermal contributions to TAE damping have essentially vanished $[9,11]$.

The scenario development presented in the present paper is performed in deuterium plasmas, in which $\alpha$-driven TAEs are absent. In order to probe the distance to the TAE instability threshold of the plasmas obtained, ions in the $\mathrm{MeV}$ range of energies are created using ICRH. In all presented pulses with ICRH power, standard hydrogen minority heating has been used. The central confinement field is $B_{0}=3.4 \mathrm{~T}$ and the ICRH frequency is set to $51 \mathrm{MHz}$, locating the fundamental resonance in the vicinity of the magnetic axis. The hydrogen concentration was in the range $\sim 2-7 \%$. It should be noted that the ICRH drive and the alpha drive differ in nature, as the corresponding fast 
ion distribution functions have different structures. For instance, because ICRH ions are typically trapped in the outboard region of the plasma, they tend to preferentially excite TAEs with a ballooned structure. Alphas, on the other hand, include a large fraction of passing particles, and can thus also excite anti-ballooned TAEs[11]. Therefore, a comparison of the required power for ICRH-driven and alpha-driven TAE excitation can only be considered as a rough estimate. Comprehensive numerical simulations, beyond the scope of the present paper, including a precise description of the fast ion populations and of the TAE mode structure must be performed to establish a quantitative correspondence between the excitation thresholds in the two cases.

Various plasma currents have been tested, in the range $I_{p}=2-3 \mathrm{MA}$. It has been established that $I_{p}=2.5 \mathrm{MA}$ constitutes a good compromise between satisfactory energetic particle confinement (which has also been shown to hold true for $3.5 \mathrm{MeV}$ alpha particles in numerical simulations) and low density operation.

\section{Internal Transport Barriers in JET-ILW}

As discussed previously, it is desirable to produce low density plasmas in order to maximise the fast hydrogen ion slowing-down time. However, since the installation of the ITER-like Wall (ILW) in JET[18], typical plasma densities are higher than those obtained with the former carbon wall (C-wall)[19]. In the present effort, it is needed to reconcile the contradictory requirements of elevated q-profile plasmas, which is typically achieved by switching-on the NBI power early in the discharge, with the requirements of obtaining low density in the peak-performance period. In Fig. 1 is plotted the line-integrated density measured by interferometry along a central chord in the peakperformance phase versus the same quantity at the NBI switch-on time. This figure was produced by selecting pulses with comparable total NBI power and $I_{p} \leq 2.75 \mathrm{MA}$. In order to remove measurement noise and slow plasma variations, $\Delta t_{\text {peak }}$ is defined as a finite time interval of at least $100 \mathrm{~ms}$ centered on the peak performance time, over which the considered quantities are averaged. The error bars in the figure result from this averaging procedure.

It is clearly observed that it is beneficial to start the plasma with as low a density as possible, while obviously still fulfilling the operational limits of the JET-ILW. This was done by implementing the so-called staggered-PINI recipe, consisting of firing the various NBI injectors with various delays depending on their respective shine-through limit and selected voltage. This recipe has allowed lower densities at the time of peak performance to be achieved compared to other pulses operated in JET-ILW. Fig. 2 shows a comparison of the density obtained versus plasma current during the peak performance phase for all deuterium pulses with $P_{N B I}>15 \mathrm{MW}$ and $I_{p}>2 \mathrm{MA}$ performed in JET since the installation of the ILW. Lines and arrows have been superimposed on the graph for each case to serve as a guide to the eye.

In order to obtain elevated values of the safety factor $q$, a common method employed in advanced scenarios is to inject external power during the current ramp-up phase 
in order to slow down the current profile diffusion process[20, 21]. To confirm the applicability of this method in JET-ILW, several similar shots have been performed, varying only the NBI switch-on time $\left(t_{N B I}\right)$. The corresponding time traces for four discharges are shown in Fig. 3, and the resulting q-profile at the time of peak performance (with $\Delta t_{\text {peak }}=500 \mathrm{~ms}$ ) reconstructed using EFIT[22] constrained with polarimetry and kinetic profiles is shown in Fig. 4.

This confirms that the variation of the NBI switch-on time is an efficient method of producing high values of the central safety factor $\left(q_{0}\right)$. Although EFIT predicts a very low shear in an extended region near the plasma center, it should be pointed out that it is often difficult to have a high accuracy of the equilibrium reconstruction in the central region. Nevertheless, except in a very limited number pulses in which Alfvén Cascades were observed, indicating a negative shear in the plasma center[23], no experimental indications of systematic q-profile reversal were obtained.

Unsurprisingly, we note that the modifications induced by varying $t_{N B I}$ are not limited to $q_{0}$. On the bottom graph of Fig. 3, it is clearly seen that the neutron rate at the time of peak performance also varies significantly from pulse to pulse. As an illustration, of these four discharges, pulse \#90189 achieves $q_{0} \approx 1.5$ with the highest neutron rate, whereas pulse \#90194 is characterised by $q_{0} \approx 2.5$, but a significantly lower neutron rate. Therefore, since both parameters are important for TAE excitation, a trade-off between $q_{0}$ and overall performance is required.

The presence of an extended central region of low positive shear is known to be prone to the triggering of ITBs when the minimal value of $q$ reaches rational values[24]. For the first time since the installation of the ILW, ITBs have been clearly observed in a number of discharges performed in the framework of this experimental effort. Fig. 5 shows time traces of pulse \#92054 with $P_{N B I} \approx 25 \mathrm{MW}$. This pulse uses only NBI power at the peak performance time of the discharge, thus fulfilling the criteria listed above for $\alpha$-driven TAE observation in DT discharges. ICRH power is applied after the time of peak performance in order to ensure a safe plasma termination.

In this discharge, it is observed that $T_{i}(0)$ and central toroidal rotation $\left(\omega_{\text {tor }}\right)$ steadily increase with time. The start time of the ITB development is consistent with the appearance of the $q=2$ surface in the plasma, although the equilibrium reconstruction is not accurate enough to reach a firm conclusion on this point. The critical energy for a D NBI ion in a $5 \mathrm{keV}$ background plasma is $E_{c} \sim 150 \mathrm{keV}$, i.e. significantly larger than the beam injection energy. As a result, in these relatively low density, high temperature discharges, NBI heating preferentially acts as an ion heating source and the electron temperature increases at a slower rate than the ion temperature. The resulting electron, ion and density profiles versus major radius in the plasma mid-plane at the times denoted by the dashed lines in Fig. 5 are shown in Fig. 6. The ion temperature profile has been produced using data from Charge Exchange Recombination Spectroscopy (CXRS) of Neon X atoms, and X-Crystal spectrometry (XCS). Electron temperature is deduced from the Electron Cyclotron Emission (ECE) radiometer and High Resolution Thomson Scattering (HRTS) systems. Electron density has been obtained from HRTS and LIDAR 
Thomson Scattering data. In all considered pulses, good consistency between the various systems installed in JET has been achieved. Therefore, in Fig. 6, ECE data and part of the HRTS data is shown for the electron temperature, whereas only HRTS data is plotted for the electron density.

The central ion temperature was found to reach $T_{i}(0) \gtrsim 10 \mathrm{keV}$ in high power discharges, whereas $T_{e}(0) \sim 5 \mathrm{keV}$. As a result of the ITB appearance, the density profile is peaked, as shown in the bottom graph of Fig. 6. Simultaneously, it is also observed that $T_{i}(0)$ and $T_{e}(0)$ start to decrease slightly from their peak values before the end of the NBI-only phase. This results from the core impurity accumulation, likely to be due to the density peaking caused by the ITB, consistently with past observations in ITB plasmas performed in JET equipped with the C-wall[25]. Impurities involved have been identified as mostly tungsten and nickel. It must be pointed out that this process does not compromise the objectives of the present effort from being reached, since the impurity accumulation is not severe enough at the time of peak performance to induce a termination of the pulse by the device protection systems.

\section{Neutron rate and performance}

A central goal of the present scenario development is to establish that sufficient fusion performance can be obtained in an extrapolated DT plasma to ensure that $\alpha$-driven TAEs can be observed, despite the requirement that no ICRH power must be used before the time of peak performance. In Fig. 7 is plotted the neutron rate at peak performance versus the total injected power, including NBI and NBI+ICRH phases for all successful pulses performed in the framework of this experiment. ICRH can contribute to the neutron rate through 1) an increase of global plasma quantities (electron and ion temperature, essentially), and 2) the generation of energetic deuterons by harmonic heating[26, 27]. According to calculations performed with the CYRANO[28] and EVE[29] codes for various pulses in which ICRH power was used up to $4.5 \mathrm{MW}$ with hydrogen concentrations $n_{H} / n_{e} \sim 2-7 \%$, second harmonic deuterium damping never exceeds $25 \%$ of the total RF power damping. Although Neutral Particle Analyzers (NPAs) do observe non-thermal deuterium ions in some pulses, the resulting deuterium acceleration appears to be insufficient to significantly contribute to the neutron rate. This is further confirmed by neutron spectroscopy analysis of these pulses[30], which shows that the $\mathrm{RF}$ contribution to the neutron rate does not exceed $15 \%$ even at low levels of hydrogen concentrations $(\lesssim 2 \%)$.

In Fig. 8 is plotted the measured neutron rate versus $n_{D} P_{N B I} \tau_{s, e}$ with $n_{D}$ the deuterium density (deduced from the electron density and $Z_{\text {eff }}$ measurement), and $\tau_{s, e}$ the electron slowing-down time. The beam-target reaction rate computed with the PENCIL code[31] using the measured kinetic profiles is found to scale linearly with this quantity, as it should[32], and to agree well with the experimental measurement for low to medium NBI power. It is observed that at high NBI power, there is a significant departure of the measured neutron rate from that calculated using PENCIL, which results 
from thermonuclear and beam-beam contributions to the neutron rate, although the latter is found not to exceed $10 \%$ in these pulses.

In order to further characterise the role of ICRH power, pulses \#92415 and \#92416 have been performed at 2.5MA, and the afterglow scheme has been tested from an operational standpoint in both. As shown in Fig. 9, the two shots are identical except for the fact that ICRH was only used for shot termination in \#92415, whereas it was applied throughout in pulse \#92416, resulting in a significant increase of the neutron rate in the latter with respect to the former. This is caused by a combination of higher ion temperature and density in the second case.

The TRANSP code[33] has been used to interpret the experimental measurements of pulse \#92416, and estimate the various contributions to the total neutron rate. The result is shown in Fig. 10. The simulated total neutron rate is in fair agreement with the experimental value during the high NBI power phase of the discharge. During the afterglow phase, the simulated value underestimates the mesured neutron rate. This is attributed to the fact that the afterglow phase is usually associated with measurement uncertainties, as some diagnostics rely on NBI to operate. The afterglow phase description will be refined in future simulations, but this slight discrepancy does not modify our conclusions. The ITB development is found to result in a significant increase of the thermonuclear contribution to the neutron rate. The benefit from this large contribution is that when the afterglow phase starts, the associated neutron rate decay occurs on the energy confinement timescale, whereas the beam-target contribution decrease is linked to the slowing-down time of NBI ions. In a DT discharge, this means that the alpha production remains substantial during a finite time following the power switch-off. The alpha decay, resulting from the combination of decreasing ion temperature and density and $3.5 \mathrm{MeV}$ alpha slowing-down, is therefore expected to occur on a timescale significantly longer than the NBI ion slowing-down time.

In pulses performed with $25 \mathrm{MW}$ of NBI power and using only NBI power before peak performance, it is found that a substantial fraction of the neutron rate comes from thermal fusion reactions. As an illustration, in the case of pulse \#92054 at $t \approx 6.6 \mathrm{~s}$, which approximately corresponds to the highest neutron rate shown in Fig. 8, TRANSP simulations indicate that the thermonuclear contribution to the total neutron rate reaches $33 \%$, whereas $61 \%$ (resp. 6\%) comes from beam-target (resp. beam-beam) reactions.

\section{TAE stability in deuterium plasmas}

The objective of ICRH power use in these deuterium pulses is to probe some of the best performing discharges with respect to TAE stability. This is what is done in the comparison between pulses \#92415 and \#92416 (Fig. 9). In pulse \#92415, no significant AE activity has been recorded by the Mirnov coils. In most discharges with RF power $\left(P_{R F} \gtrsim 2 \mathrm{MW}\right)$ on the other hand, TAEs are observed in the range 100-200kHz. As shown in Fig. 11, this is the case in pulse \#92416 with ICRH power throughout. 
In this discharge, it is observed that TAEs are present until $t \approx 5.3 \mathrm{~s}$. At this time, the NBI power is increased from $10 \mathrm{MW}$ to $25 \mathrm{MW}$. The TAE frequency shifts upwards

as a result of the toroidal velocity increase. At $t \approx 5.5 \mathrm{~s}$, it starts to shift downwards following the decrease of $v_{A} \propto 1 / n_{e}^{1 / 2}$ caused by the density increase induced by the NBI fuelling. At $t \approx 5.6 \mathrm{~s}$, the TAEs disappear due to the damping by NBI ions despite the presence of $5 \mathrm{MW}$ of ICRH power. The afterglow phase starts at $t=6.1$ s and is followed by the reappearance of the TAEs with a delay $\sim 100 \mathrm{~ms}$, which is consistent with the deuterium ion slowing-down.

Fig. 12 shows a zoom on the TAEs reappearing at $t \approx 6.2 \mathrm{~s}$. The toroidal array of Mirnov coils installed on JET allows the toroidal mode numbers $(n)$ to be estimated. It shows that the corresponding TAEs are characterised by $n=4,5$ and 6 . Also visible on this figure is the signal from the active antenna used for TAE excitation in JET[34]. Although this system has also been used in these discharges, the results obtained will be reported in a separate article.

In order to further characterise these modes, the HELENA[35] and MISHKA[36] codes have been run using the data from EFIT at $t=6.2 \mathrm{~s}$ as input. The result from the MISHKA calculation corresponding to the mode $n=5$ is displayed in Fig. 13. The plotted quantity on this figure is the radial contravariant component of the fluid velocity $V^{1}$ (see Ref. [36]), which is representative of the mode structure. It shows a TAE located at normalised radius $\rho \approx 0.4$ dominated by poloidal components $m=10$ and $m=11$. It is consistent with experimental data, although the MHD diagnostics in JET do not allow a precise radial localisation of the modes to be performed. Nevertheless, reflectometry data do not indicate the presence of any TAEs in the outer parts of the plasma at this time of the discharge, which is consistent with the presence of a core-localised TAE.

Finally, it must be noted that the linear MHD calculations performed with MISHKA display a large sensitivity with respect to the precise value of $q_{0}$, as a result of the very low central shear predicted by EFIT. Experimentally, however, TAEs are commonly observed in discharges with ICRH power, and appear to be quite robust with respect to changes in the plasma parameters, including $q$. This could be an indication of the fact that the q-profile is not as flat as predicted. Non-linear MHD calculations, including sensitivity studies, in the presence of energetic hydrogen ions retaining finite orbit effects performed with the SELFO[37] and VENUS[38] codes are ongoing and will be the subject of a future publication.

\section{Predictions for deuterium-tritium discharges}

In order to establish the relevance of the developed scenario aimed at $\alpha$-driven TAE observation, it is necessary to perform extrapolations of these deuterium pulses to a DT mixture. This has been achieved by carrying out several runs with the TRANSP code. The reference pulse used for these calculations is \#92054, using NBI only in its peak performance phase, and already presented in section 3. The first step is to carefully interpret the measurements by using the experimental values of $T_{i}, T_{e}, n_{e}$ and $\omega_{t o r}$. The 
measured effective charge $\left(Z_{e f f}\right)$ was used as well, assuming the $Z_{\text {eff }}$ profile was flat. At this stage, the main source of uncertainty corresponds to the impurity content of the discharge. Several hypotheses have been tested in this domain, with the objective of reproducing the plasma energy $W_{p}$, neutron rate $R_{N T}$ and radiated power $P_{\text {rad }}$ :

(i) When the only impurity is assumed to be tungsten (W) and $T_{i}$ is taken to be the experimental value, $W_{p}$ is correctly reproduced. On the other hand, both $R_{N T}$ and $P_{\text {rad }}$ are overestimated.

(ii) When the only impurity is assumed to be beryllium (Be), $W_{p}$ is well recovered, but $R_{N T}$ is significantly underestimated as $Z_{\text {eff }}$ increases, pointing to an excessive dilution with respect to the experiment. $P_{\text {rad }}$ is much too low compared to experimental bolometry measurements.

(iii) When the only impurity is assumed to be $\mathrm{W}$ but $T_{i}$ is chosen to be the lower range of error bars of CXRS measurements, it is possible to reproduce $W_{p}$ and $P_{\text {rad }}$ with good accuracy. $R_{N T}$ is also correctly recovered, with only a slight underestimation at the time of peak performance.

These results are summarised in Fig. 14. The outcome of these simulations is that the actual plasma probably contains a mix of $\mathrm{W}$ and $\mathrm{Be}$ (as well as other light impurities). Given the uncertainties in this domain, the DT extrapolation has been performed by assuming that $\mathrm{W}$ was the only impurity, but that $T_{i}$ had a finite range of uncertainty corresponding to options 1 and 3 above.

This pulse has been used as a basis for extrapolation, replacing both fuel and NBI deuterium ions by a 50\%-50\% mixture of deuterium and tritium. This has allowed the expected alpha density, pressure and corresponding $\beta_{T \alpha}$ to be estimated. The result obtained is shown in Fig. 15. As discussed previously, the finite range of $T_{i}$ provides a range of $\beta_{T \alpha}$ values. It turns out that the $\beta_{T \alpha}$ obtained is comparable to, or even slightly larger than, the value achieved in successful TFTR experiments[9]. Although this prediction cannot replace comprehensive simulations that take into account bulk plasma transport, MHD and fast particle effects, it encourages us to develop further this scenario in view of future DT campaigns in JET.

\section{Conclusions}

A significant effort has been invested in the development of a scenario for the observation of $\alpha$-driven TAEs in an upcoming DT campaign of JET. With this end in mind, deuterium discharges have been performed at comparatively low densities, elevated qprofiles and using only NBI power prior to the peak performance phase of the pulse. The selected plasma current is $I_{p}=2.5 \mathrm{MA}$, which has been found to constitute a good compromise between low density operation and satisfactory energetic particle confinement. The magnetic field $B_{T}=3.4 \mathrm{~T}$ allows ICRH to be used for TAE probing purposes by locating the fundamental hydrogen cyclotron layer close to the magnetic axis thereby generating a significant population of well-confined energetic hydrogen 
ions. With these parameters, ITBs have been unambiguously observed for the first time since the installation of the ILW in JET. They have allowed the central ion temperature to reach $T_{i} \gtrsim 13 \mathrm{keV}$ with $25 \mathrm{MW}$ of NBI power, resulting in a neutron rate $R_{N T} \gtrsim 1.2 \times 10^{16} / \mathrm{s}$, with a significant thermonuclear contribution (up to $\sim 40 \%$ ). By using ICRH, TAEs have been routinely observed in the range $100-200 \mathrm{kHz}$ when $P_{I C R H} \gtrsim 1-2 \mathrm{MW}$. They have been observed to be damped at high NBI power. Linear MHD calculations and the absence of any edge TAEs on reflectometry measurements for these discharges show that these modes are core-localised. Interpretative integrated simulations of the best performing discharges have been performed using various hypotheses in terms of impurity content, and then extrapolated to DT plasmas. They predict that normalised alpha pressure values could be comparable or even slightly larger than values measured in successful $\alpha$-driven TAE experiments in TFTR. We plan to develop this scenario further by performing discharges at higher NBI power in an upcoming deuterium campaign, and also consolidate extrapolations to DT by performing similar pulses in pure tritium plasmas before applying this scenario to the next JET DT campaign.

\section{Acknowledgments}

This work has been carried out within the framework of the EUROfusion Consortium and has received funding from the Euratom research and training programme 20142018 under grant agreement No 633053 and from the RCUK Energy Programme [grant number EP/P012450/1]. The views and opinions expressed herein do not necessarily reflect those of the European Commission. Support for the US group was provided by the US DOE under Grant Number DE-FG02-99ER54563. This work was supported in part by the Swiss National Science Foundation, and was granted access to the HPC resources of TGCC under the allocation 2017-A0010506088 made by GENCI.

\section{References}

[1] C. Z. Cheng and M. S. Chance. Low-n shear alfvén spectra in axisymmetric toroidal plasmas. The Physics of Fluids, 29(11):3695-3701, 1986.

[2] King-Lap Wong. A review of alfvén eigenmode observations in toroidal plasmas. Plasma Physics and Controlled Fusion, 41(1):R1, 1999.

[3] N.N. Gorelenkov, S.D. Pinches, and K. Toi. Energetic particle physics in fusion research in preparation for burning plasma experiments. Nuclear Fusion, 54(12):125001, 2014.

[4] S. D. Pinches, I. T. Chapman, Ph. W. Lauber, H. J. C. Oliver, S. E. Sharapov, K. Shinohara, and K. Tani. Energetic ions in iter plasmas. Physics of Plasmas, 22(2):021807, 2015.

[5] M Schneller, Ph Lauber, and S Briguglio. Nonlinear energetic particle transport in the presence of multiple alfvnic waves in iter. Plasma Physics and Controlled Fusion, 58(1):014019, 2016.

[6] M. Fitzgerald, S.E. Sharapov, P. Rodrigues, and D. Borba. Predictive nonlinear studies of tae-induced alpha-particle transport in the $\mathrm{q}=10$ iter baseline scenario. Nuclear Fusion, 56(11):112010, 2016.

[7] R.V. Budny, M.G. Bell, H. Biglari, M. Bitter, C.E. Bush, C.Z. Cheng, E.D. Fredrickson, B. Grek, K.W. Hill, H. Hsuan, A.C. Janos, D.L. Jassby, D.W. Johnson, L.C. Johnson, B. LeBlanc, D.C. 
Scenario development for the observation of alpha-driven instabilities in JET DT plasmas12

McCune, D.R. Mikkelsen, H.K.Park, A.T. Ramsey, S.A. Sabbagh, S.D. Scott, J.F. Schivell, J.D. Strachan, B.C. Stratton, E.J. Synakowski, G. Taylor, M.C. Zarnstorff, and S.J. Zweben. Simulations of deuterium-tritium experiments in tftr. Nuclear Fusion, 32(3):429, 1992.

[8] D.A. Spong, C.L. Hedrick, and B.A. Carreras. Strategies for modifying alpha driven tae thresholds through q profile and ion temperature control. Nuclear Fusion, 35(12):1687, 1995.

[9] R. Nazikian, G. Y. Fu, S. H. Batha, M. G. Bell, R. E. Bell, R. V. Budny, C. E. Bush, Z. Chang, Y. Chen, C. Z. Cheng, D. S. Darrow, P. C. Efthimion, E. D. Fredrickson, N. N. Gorelenkov, B. Leblanc, F. M. Levinton, R. Majeski, E. Mazzucato, S. S. Medley, H. K. Park, M. P. Petrov, D. A. Spong, J. D. Strachan, E. J. Synakowski, G. Taylor, S. Von Goeler, R. B. White, K. L. Wong, and S. J. Zweben. Alpha-particle-driven toroidal alfvén eigenmodes in the tokamak fusion test reactor. Phys. Rev. Lett., 78:2976-2979, Apr 1997.

[10] S.J. Zweben, R.V. Budny, D.S. Darrow, S.S. Medley, R. Nazikian, B.C. Stratton, E.J. Synakowski, and G. Taylor for the TFTR Group. Alpha particle physics experiments in the tokamak fusion test reactor. Nuclear Fusion, 40(1):91, 2000.

[11] S.E. Sharapov, D. Borba, A. Fasoli, W. Kerner, L.-G. Eriksson, R.F. Heeter, G.T.A. Huysmans, and M.J. Mantsinen. Stability of alpha particle driven alfvén eigenmodes in high performance jet dt plasmas. Nuclear Fusion, 39(3):373, 1999.

[12] S.E. Sharapov, B. Alper, D. Borba, L.-G. Eriksson, A. Fasoli, R.D. Gill, A. Gondhalekar, C. Gormezano, R.F. Heeter, G.T.A. Huysmans, J. Jacquinot, A.A. Korotkov, P. Lamalle, M.J. Mantsinen, D.C. McDonald, F.G. Rimini, D.F.H. Start, D. Testa, P.R. Thomas, and JET Team. Energetic particle physics in jet. Nuclear Fusion, 40(7):1363, 2000.

[13] S. E. Sharapov, L.-G. Eriksson, A. Fasoli, G. Gorini, J. Kllne, V. G. Kiptily, A. A. Korotkov, A. Murari, S. D. Pinches, D. S. Testa, and P. R. Thomas. Chapter 5: Burning plasma studies at jet. Fusion Science and Technology, 53(4):989-1022, 2008.

[14] G. Y. Fu and J. W. Van Dam. Excitation of the toroidicityinduced shear alfvén eigenmode by fusion alpha particles in an ignited tokamak. Physics of Fluids B: Plasma Physics, 1(10):1949$1952,1989$.

[15] C. Z. Cheng. Alpha particle destabilization of the toroidicityinduced alfvén eigenmodes. Physics of Fluids B: Plasma Physics, 3(9):2463-2471, 1991.

[16] B N Breizman and S E Sharapov. Energetic particle drive for toroidicity-induced alfven eigenmodes and kinetic toroidicity-induced alfven eigenmodes in a low-shear tokamak. Plasma Physics and Controlled Fusion, 37(10):1057, 1995.

[17] M. Cox and D.F.H. Start. A 2-d fokker-planck treatment of the icrf enhancement of beam-driven currents. Nuclear Fusion, 24(4):399, 1984.

[18] F. Romanelli and JET EFDA Contributors. Overview of the jet results with the iter-like wall. Nuclear Fusion, 53(10):104002, 2013.

[19] E. Joffrin, M. Baruzzo, M. Beurskens, C. Bourdelle, S. Brezinsek, J. Bucalossi, P. Buratti, G. Calabro, C.D. Challis, M. Clever, J. Coenen, E. Delabie, R. Dux, P. Lomas, E. de la Luna, P. de Vries, J. Flanagan, L. Frassinetti, D. Frigione, C. Giroud, M. Groth, N. Hawkes, J. Hobirk, M. Lehnen, G. Maddison, J. Mailloux, C.F. Maggi, G. Matthews, M. Mayoral, A. Meigs, R. Neu, I. Nunes, T. Puetterich, F. Rimini, M. Sertoli, B. Sieglin, A.C.C. Sips, G. van Rooij, I. Voitsekhovitch, and JET-EFDA Contributors. First scenario development with the jet new iter-like wall. Nuclear Fusion, 54(1):013011, 2014.

[20] C D Challis, Yu F Baranov, G D Conway, C Gormezano, C W Gowers, N C Hawkes, T C Hender, E Joffrin, J Mailloux, D Mazon, S Podda, R Prentice, F G Rimini, S E Sharapov, A C C Sips, B C Stratton, D Testa, and K-D Zastrow. Effect of q-profile modification by lhcd on internal transport barriers. Plasma Physics and Controlled Fusion, 43(7):861, 2001.

[21] C. Gormezano, C. D. Challis, E. Joffrin, X. Litaudon, and A. C. C. Sips. Chapter 4: Advanced tokamak scenario development at jet. Fusion Science and Technology, 53(4):958-988, 2008.

[22] D.P. O'Brien, L.L. Lao, E.R. Solano, M. Garribba, T.S. Taylor, J.G. Cordey, and J.J. Ellis. Equilibrium analysis of iron core tokamaks using a full domain method. Nuclear Fusion, 
Scenario development for the observation of alpha-driven instabilities in JET DT plasmas13

32(8):1351, 1992.

[23] S. E. Sharapov, B. Alper, H. L. Berk, D. N. Borba, B. N. Breizman, C. D. Challis, A. Fasoli, N. C. Hawkes, T. C. Hender, J. Mailloux, S. D. Pinches, D. Testa, and EFDAJET work programme. Alfvn wave cascades in a tokamak. Physics of Plasmas, 9(5):2027-2036, 2002.

[24] E. Joffrin, C.D. Challis, G.D. Conway, X. Garbet, A. Gude, S. Günter, N.C. Hawkes, T.C. Hender, D.F. Howell, G.T.A. Huysmans, E. Lazzaro, P. Maget, M. Marachek, A.G. Peeters, S.D. Pinches, S.E. Sharapov, and JET-EFDA contributors. Internal transport barrier triggering by rational magnetic flux surfaces in tokamaks. Nuclear Fusion, 43(10):1167, 2003.

[25] R. Dux, C. Giroud, K.-D. Zastrow, and JET EFDA contributors. Impurity transport in internal transport barrier discharges on jet. Nuclear Fusion, 44(2):260, 2004.

[26] J. Eriksson, M. Nocente, F. Binda, C. Cazzaniga, S. Conroy, G. Ericsson, L. Giacomelli, G. Gorini, C. Hellesen, T. Hellsten, A. Hjalmarsson, A. S. Jacobsen, T. Johnson, V. Kiptily, T. Koskela, M. Mantsinen, M. Salewski, M. Schneider, S. Sharapov, M. Skiba, M. Tardocchi, M. Weiszflog, and JET Contributors. Dual sightline measurements of mev range deuterons with neutron and gamma-ray spectroscopy at jet. Nuclear Fusion, 55(12):123026, 2015.

[27] M. Schneider, T. Johnson, R. Dumont, J. Eriksson, L.-G. Eriksson, L. Giacomelli, J.-B. Girardo, T. Hellsten, E. Khilkevitch, V.G. Kiptily, T. Koskela, M. Mantsinen, M. Nocente, M. Salewski, S. E. Sharapov, A. E. Shevelev, and JET Contributors. Modelling third harmonic ion cyclotron acceleration of deuterium beams for jet fusion product studies experiments. Nuclear Fusion, 56(11):112022, 2016.

[28] E Lerche, D Van Eester, A Krasilnikov, J Ongena, P Lamalle, and JET-EFDA contributors. Modelling of d majority icrh at jet: impact of absorption at the doppler-shifted resonance. Plasma Physics and Controlled Fusion, 51(4):044006, 2009.

[29] R. J. Dumont and D. Zarzoso. Heating and current drive by ion cyclotron waves in the activated phase of iter. Nuclear Fusion, 53(1):013002, 2013.

[30] M. Gatu Johnson, L. Giacomelli, A. Hjalmarsson, M. Weiszflog, E. Andersson Sundén, S. Conroy, G. Ericsson, C. Hellesen, J. Källne, E. Ronchi, H. Sjöstrand, G. Gorini, M. Tardocchi, A. Murari, S. Popovichev, J. Sousa, R. C. Pereira, A. Combo, N. Cruz, and JET EFDA Contributors. The tofor neutron spectrometer and its first use at jet. Review of Scientific Instruments, $77(10): 10 \mathrm{E} 702,2006$.

[31] C.D. Challis, J.G. Cordey, H. Hamnén, P.M. Stubberfield, J.P. Christiansen, E. Lazzaro, D.G. Muir, D. Stork, and E. Thompson. Non-inductively driven currents in jet. Nuclear Fusion, 29(4):563, 1989.

[32] J.D. Strachan, M.G. Bell, M. Bitter, R.V. Budny, R.J. Hawryluk, K.W. Hill, H. Hsuan, D.L. Jassby, L.C. Johnson, B. LeBlanc, D.K. Mansfield, E.S. Marmar, D.M. Meade, D.R. Mikkelsen, D. Mueller, H.K. Park, A.T. Ramsey, S.D. Scott, J.A. Snipes, E.J. Synakowski, G. Taylor, and J.L. Terry. Neutron emission from tftr supershots. Nuclear Fusion, 33(7):991, 1993.

[33] R.J Goldston, D.C McCune, H.H Towner, S.L Davis, R.J Hawryluk, and G.L Schmidt. New techniques for calculating heat and particle source rates due to neutral beam injection in axisymmetric tokamaks. Journal of Computational Physics, 43(1):61 - 78, 1981.

[34] P. Puglia, W. Pires de Sa, P. Blanchard, S. Dorling, S. Dowson, A. Fasoli, J. Figueiredo, R. Galvo, M. Graham, G. Jones, C. Perez von Thun, M. Porkolab, L. Ruchko, D. Testa, P. Woskov, M.A. Albarracin-Manrique, and JET Contributors. The upgraded jet toroidal alfvn eigenmode diagnostic system. Nuclear Fusion, 56(11):112020, 2016.

[35] G.T.A. HUYSMANS, J.P. GOEDBLOED, and W. KERNER. Isoparametric bicubic hermite elements for solution of the grad-shafranov equation. International Journal of Modern Physics $C, 02(01): 371-376,1991$.

[36] A. B. Mikhailovskii, G. T. A. Huysmans, S. E. Sharapov, and W. Kerner. Optimization of computational mhd normal-mode analysis for tokamaks. Plasma Phys. Rep., 23:844, 1997.

[37] J. Hedin, T. Hellsten, L.-G. Eriksson, and T. Johnson. The influence of finite drift orbit width on icrf heating in toroidal plasmas. Nuclear Fusion, 42(5):527, 2002. 
[38] M Jucker, J P Graves, W A Cooper, and T Johnson. Ion cyclotron resonance heating with consistent finite orbit widths and anisotropic equilibria. Plasma Physics and Controlled Fusion, 53(5):054010, 2011. 


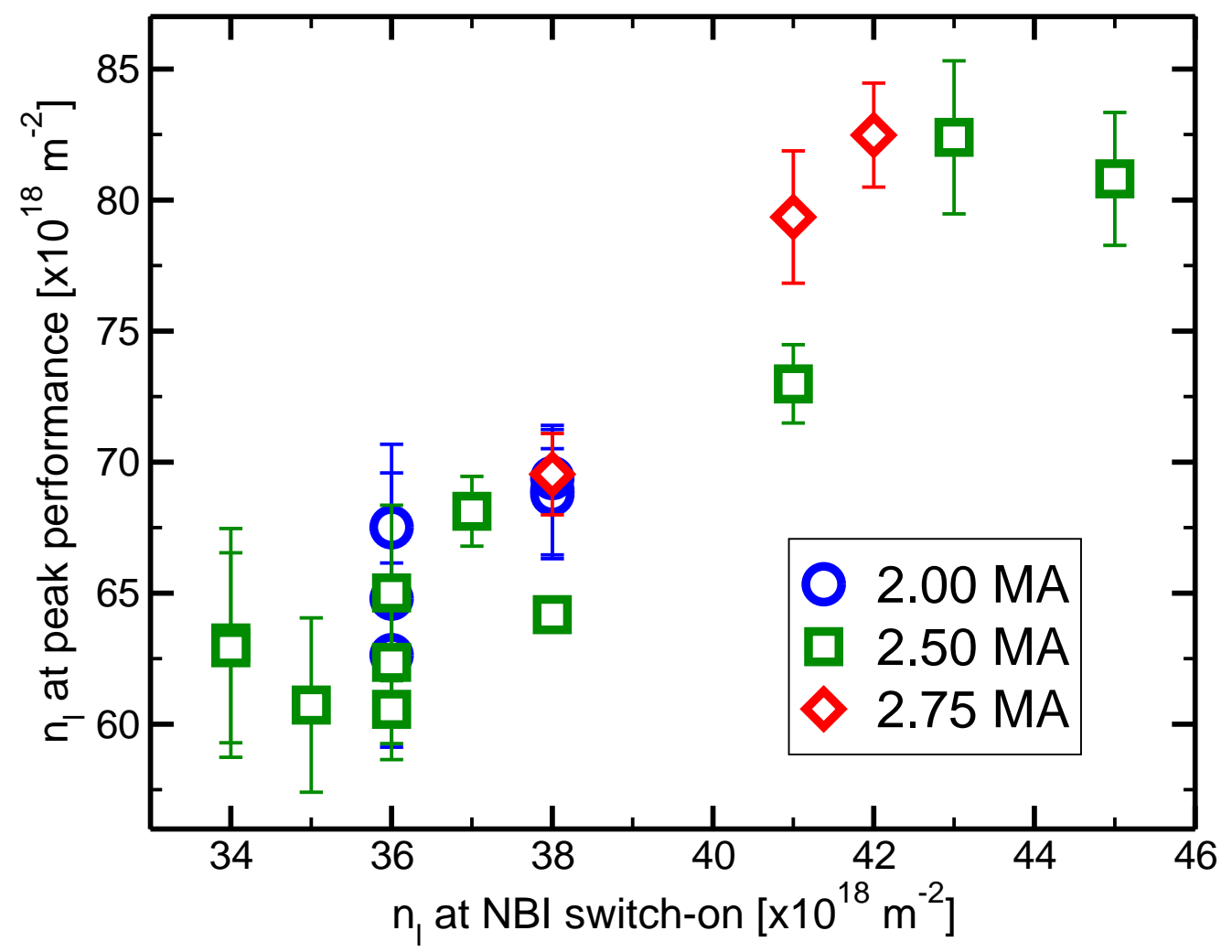

Figure 1. Line-integrated density at peak performance time versus line-integrated density at NBI switch-on time measured along a central interferometer chord, for shots with current plasma 2MA (circles), 2.5MA (squares) and 2.75MA (diamonds). 


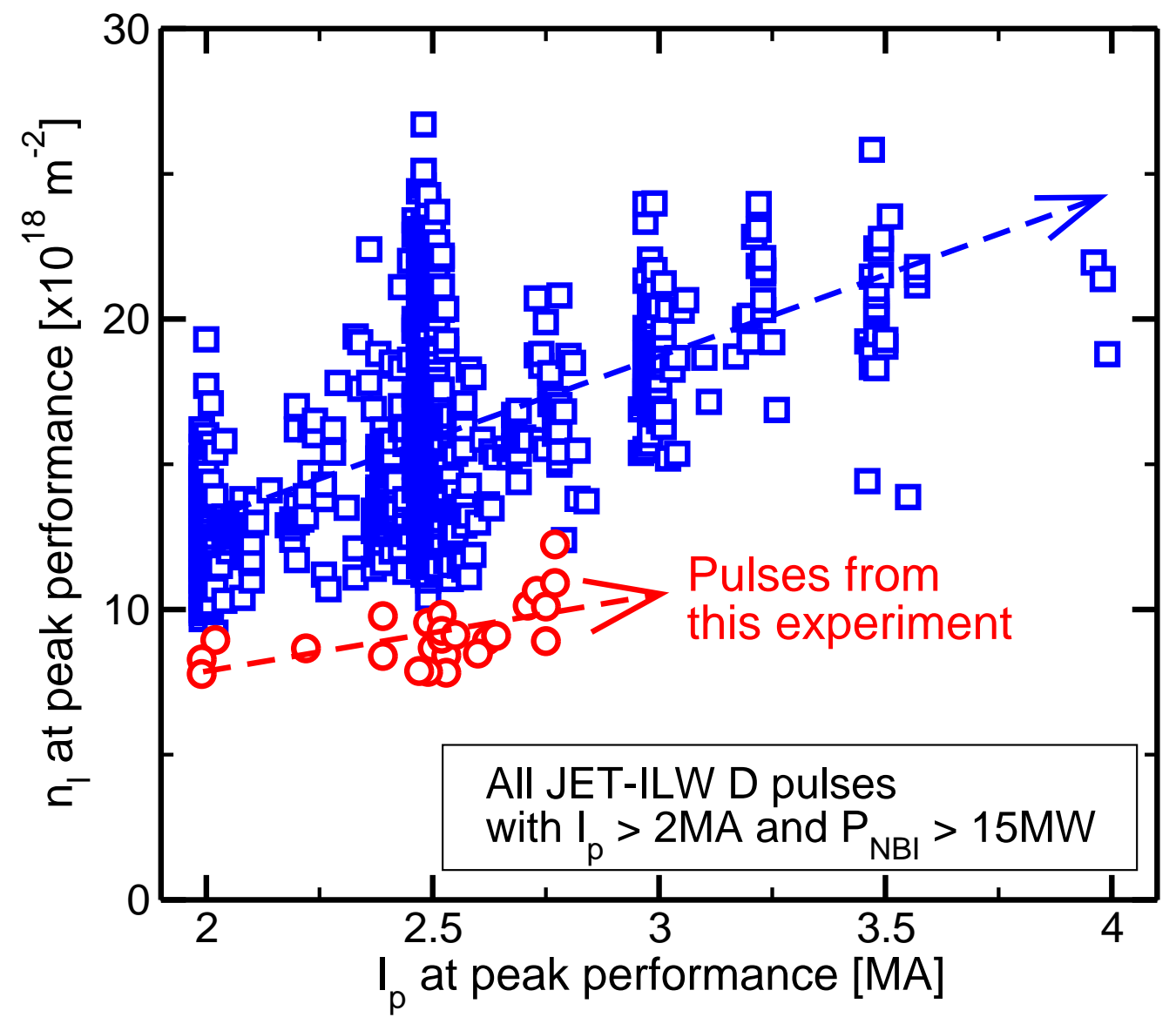

Figure 2. Line-integrated density versus current at peak performance in all deuterium pulses with $I_{p}>2 \mathrm{MA}$ and $P_{N B I}>15 \mathrm{MW}$ since the installation of the ITER-like wall in JET. The discharges discussed in this article appear as red circles, whereas all other pulses are shown as blue squares. Linear fits of the plotted datapoints (dashed lines and arrows) have been superimposed to serve as a guide to the eye for pulses performed in the framework of this experiment (red), and in all other experiments (blue). 


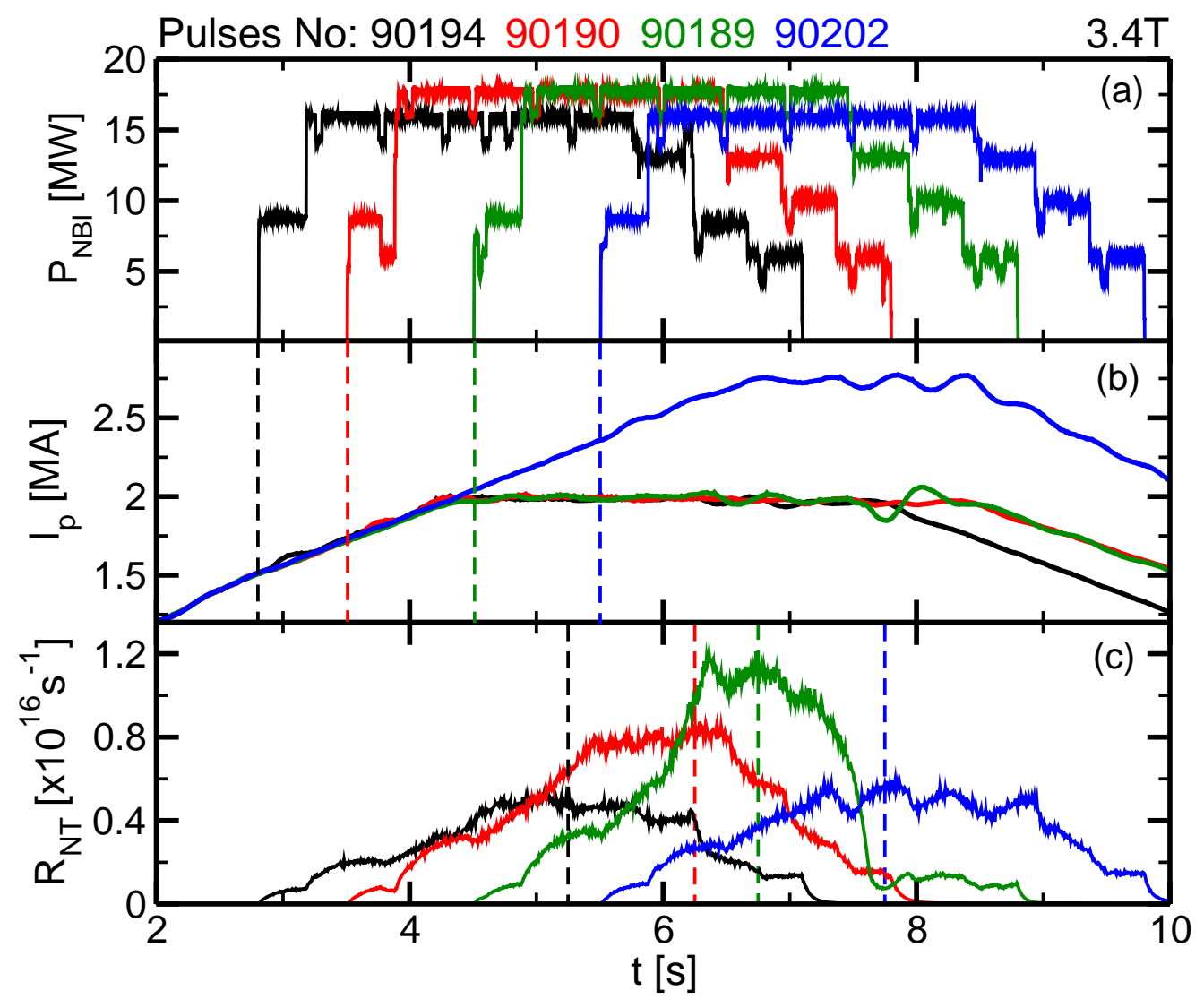

Figure 3. Comparison of pulses \#90194, \#90190,\#90189, \#90202, which differ by the time at which the NBI power is applied. (a) NBI power; (b) plasma current; (c) neutron rate. On (b), the vertical dashed lines show the NBI switch-on time. On (c), the vertical dashed lines denote the center of each peak performance period (for these discharges, $\Delta t_{\text {peak }}=500 \mathrm{~ms}$ ), and refer to Fig. 4 . 
Scenario development for the observation of alpha-driven instabilities in JET DT plasmas18

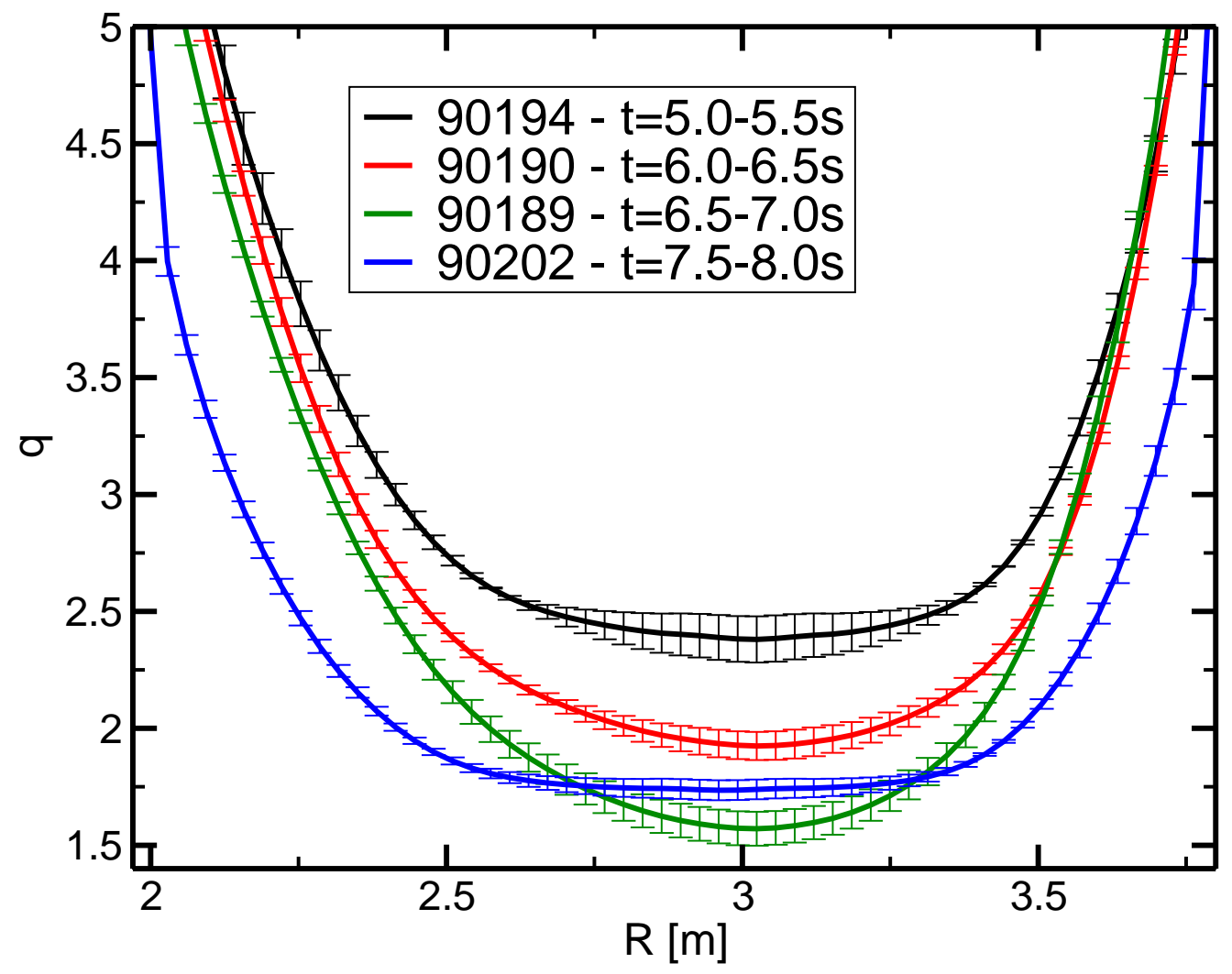

Figure 4. Safety factor profile at time of peak performance for pulses \#90194, \#90190,\#90189, \#90202 (see Fig. 3). The error bars result from the finite extent of the peak performance period. 
Scenario development for the observation of alpha-driven instabilities in JET DT plasmas19

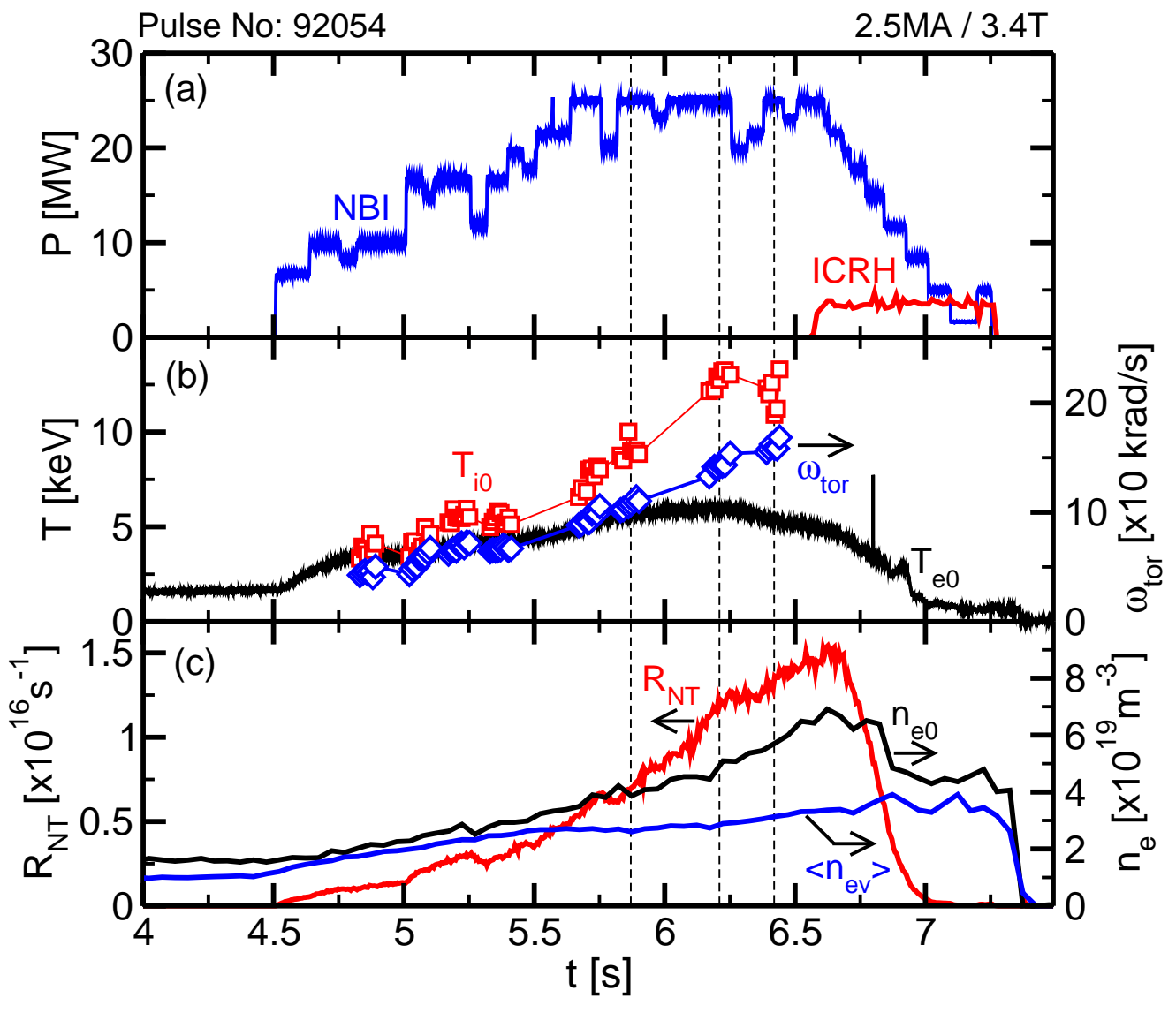

Figure 5. Pulse \#92054. (a) Auxiliary power; (b) Central electron and ion temperatures (left y-axis) and toroidal angular rotation rate (right y-axis); (c) Neutron rate (left y-axis); central and volume-averaged electron density (right y-axis). The vertical dashed lines refer to the profiles shown in Fig. 6 . 


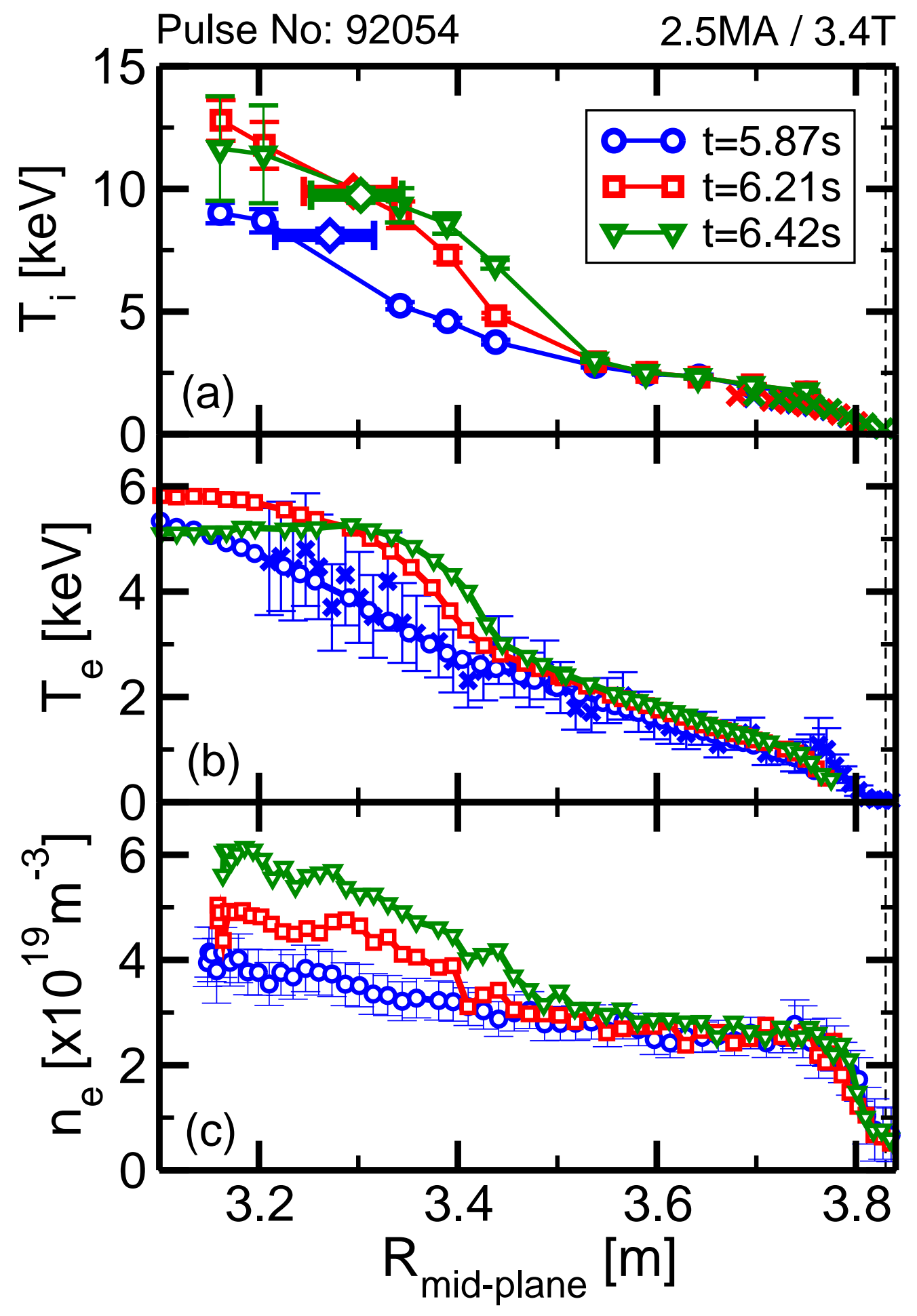

Figure 6. Pulse \#92054. (a) Ion temperature from core CXRS (open symbols, solid line and error bars) and edge CXRS (crosses) diagnostics. Also shown is the XCS data (diamonds); (b) Electron temperature from ECE radiometer (open symbols and solid lines) and HRTS system at $t=5.87 \mathrm{~s}$ and $R>3.2 \mathrm{~m}$ (crosses with error bars); (c) Electron density from HRTS system. For clarity, the corresponding error bars are only shown for the profile at $t=5.87 \mathrm{~s}$. The $\mathrm{x}$-axis corresponds to the major radius in the plasma mid-plane. The magnetic center is located at $R_{m a g} \approx 3.0 \mathrm{~m}$, whereas the separatrix is at $R_{\text {sep }} \approx 3.83 \mathrm{~m}$ (dashed line). 
Scenario development for the observation of alpha-driven instabilities in JET DT plasmas21

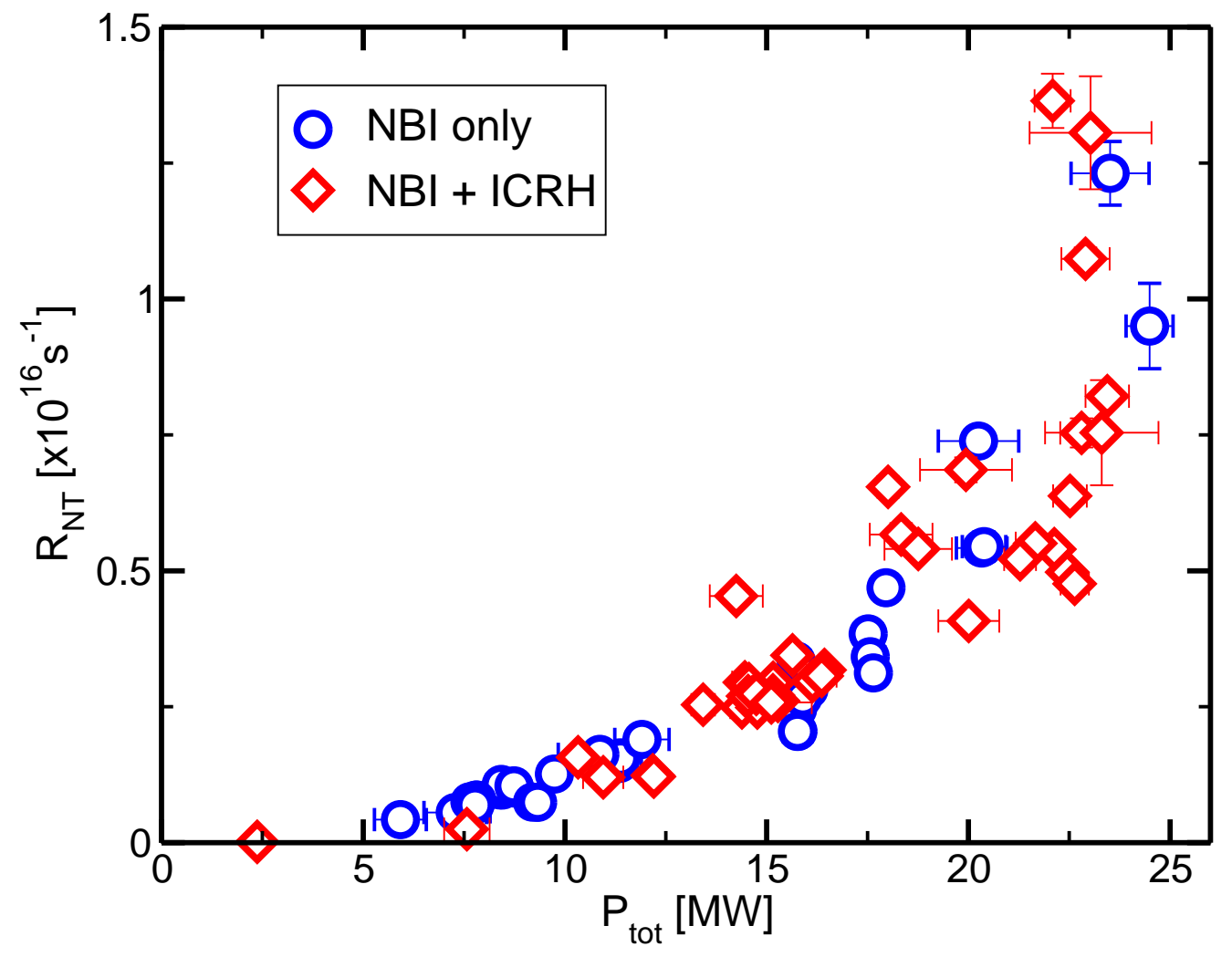

Figure 7. Neutron rate versus total (NBI+RF) power during high-performance phase, including NBI only (circles) and NBI+ICRH (diamonds) phases. 
Scenario development for the observation of alpha-driven instabilities in JET DT plasmas22

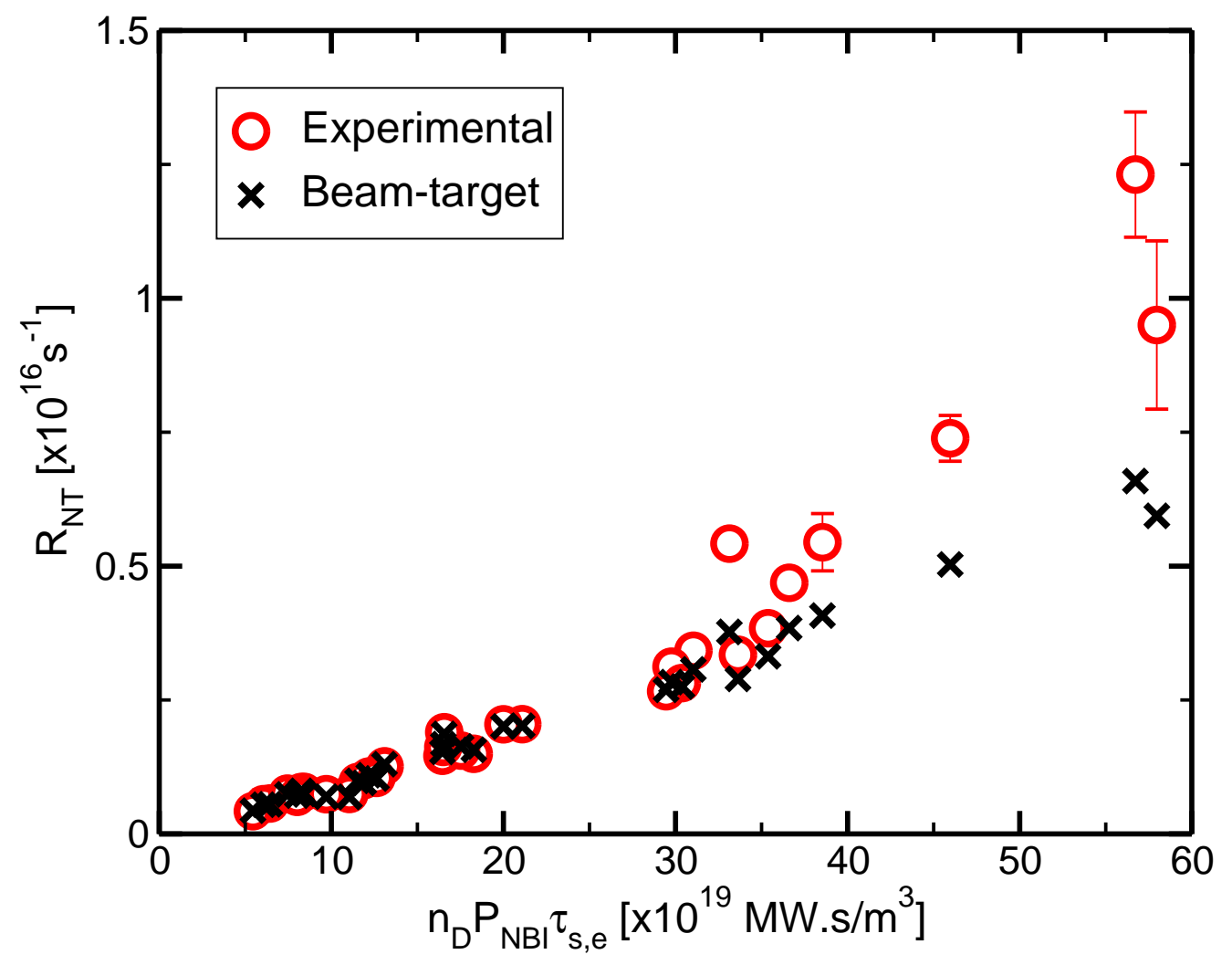

Figure 8. Measured neutron rate (circles) and beam-target neutron rate from PENCIL (crosses) versus $n_{D} P_{N B I} \tau_{s, e}$ at time of peak performance (NBI only pulses). 
Scenario development for the observation of alpha-driven instabilities in JET DT plasmas23

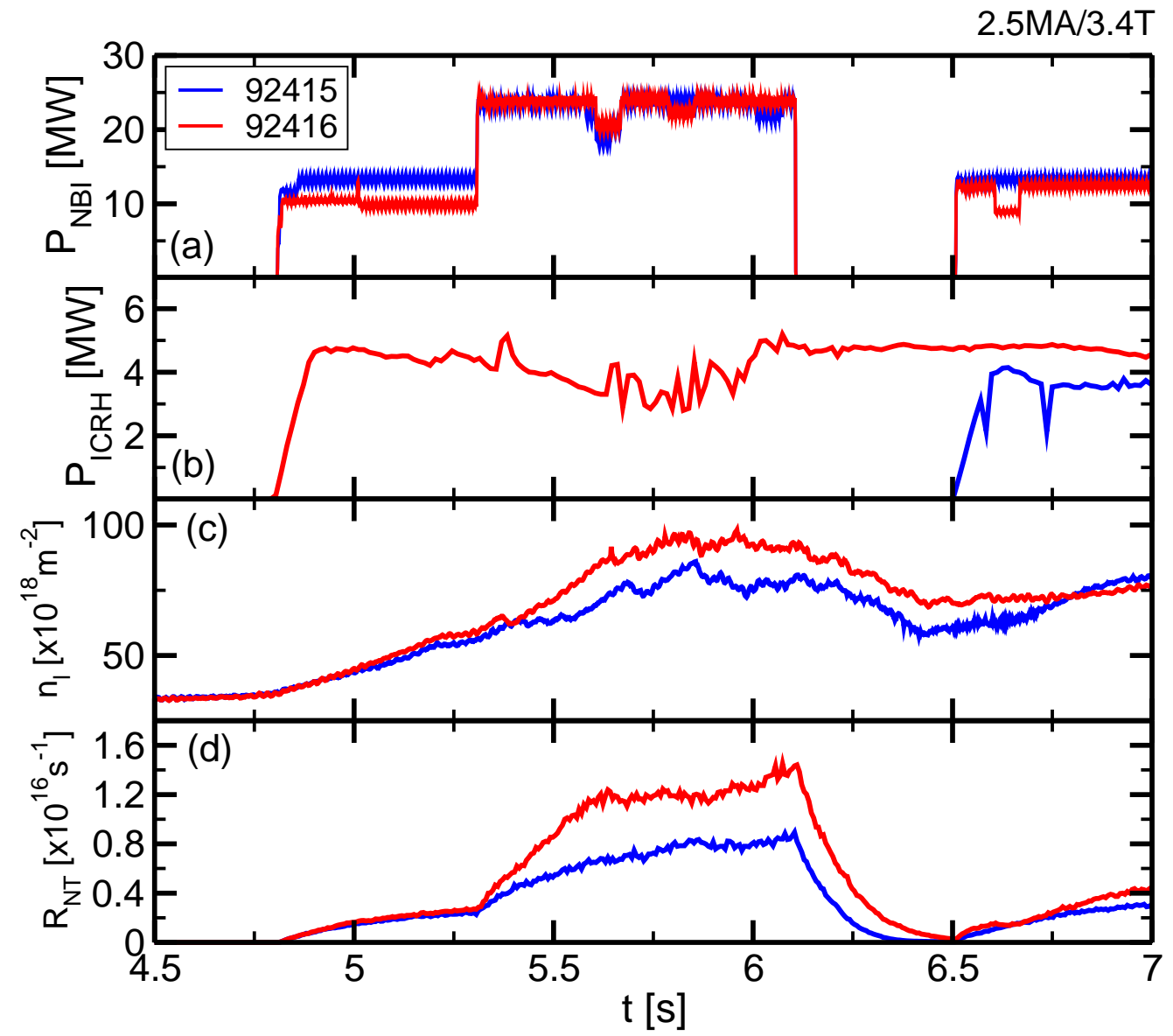

Figure 9. Comparison between pulses \#92415 and \#92416. (a) NBI power; (b) ICRH power; (c) Line-integrated density; (d) Neutron rate. 
Scenario development for the observation of alpha-driven instabilities in JET DT plasmas 24

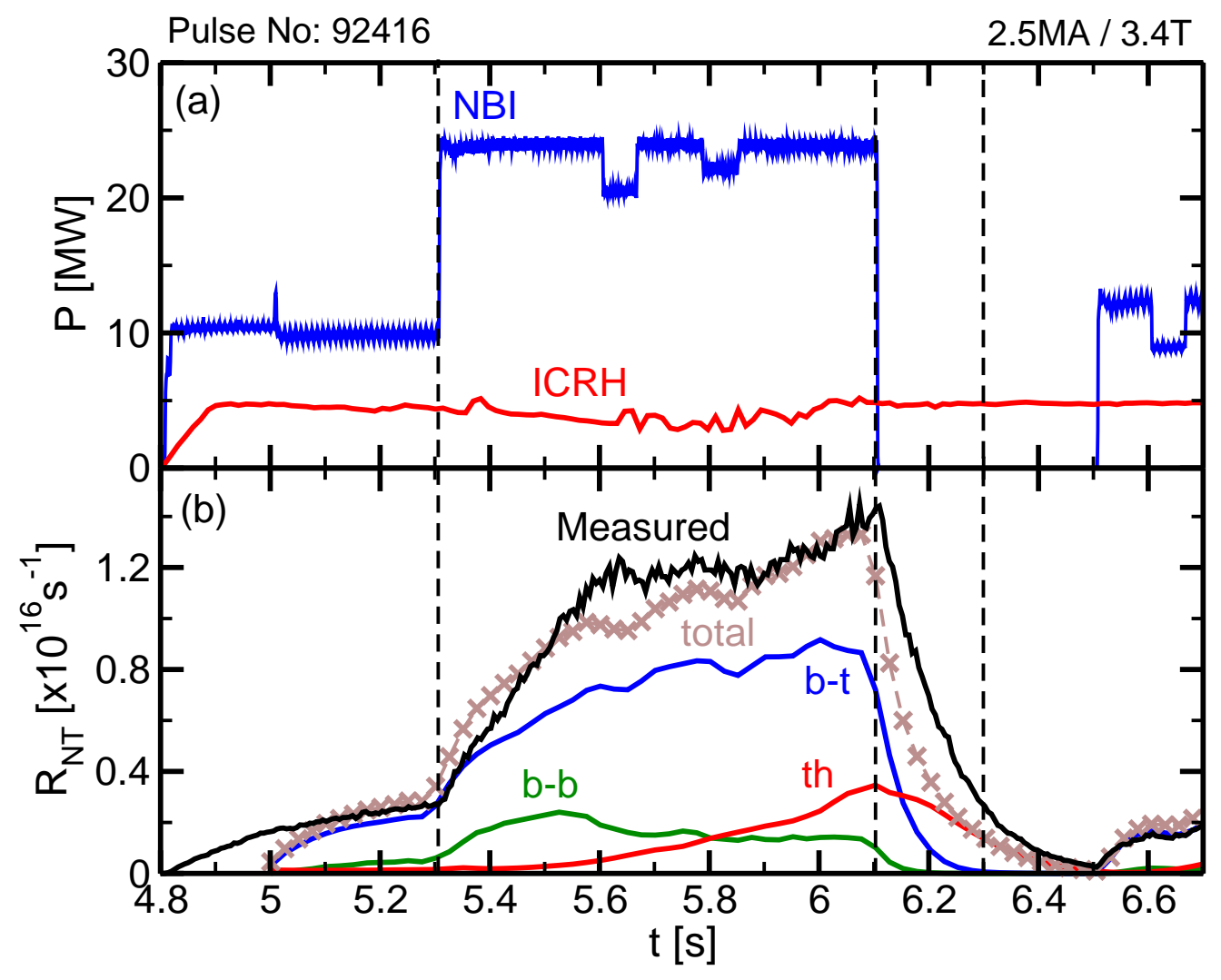

Figure 10. Pulse \#92416. (a) NBI and ICRH power; (b) Neutron rate, measured (black line); beam-beam (b-b), beam-thermal (b-t), thermonuclear (th) and total contributions computed by TRANSP. The vertical dashed lines refer to Fig. 11. 
Scenario development for the observation of alpha-driven instabilities in JET DT plasmas25

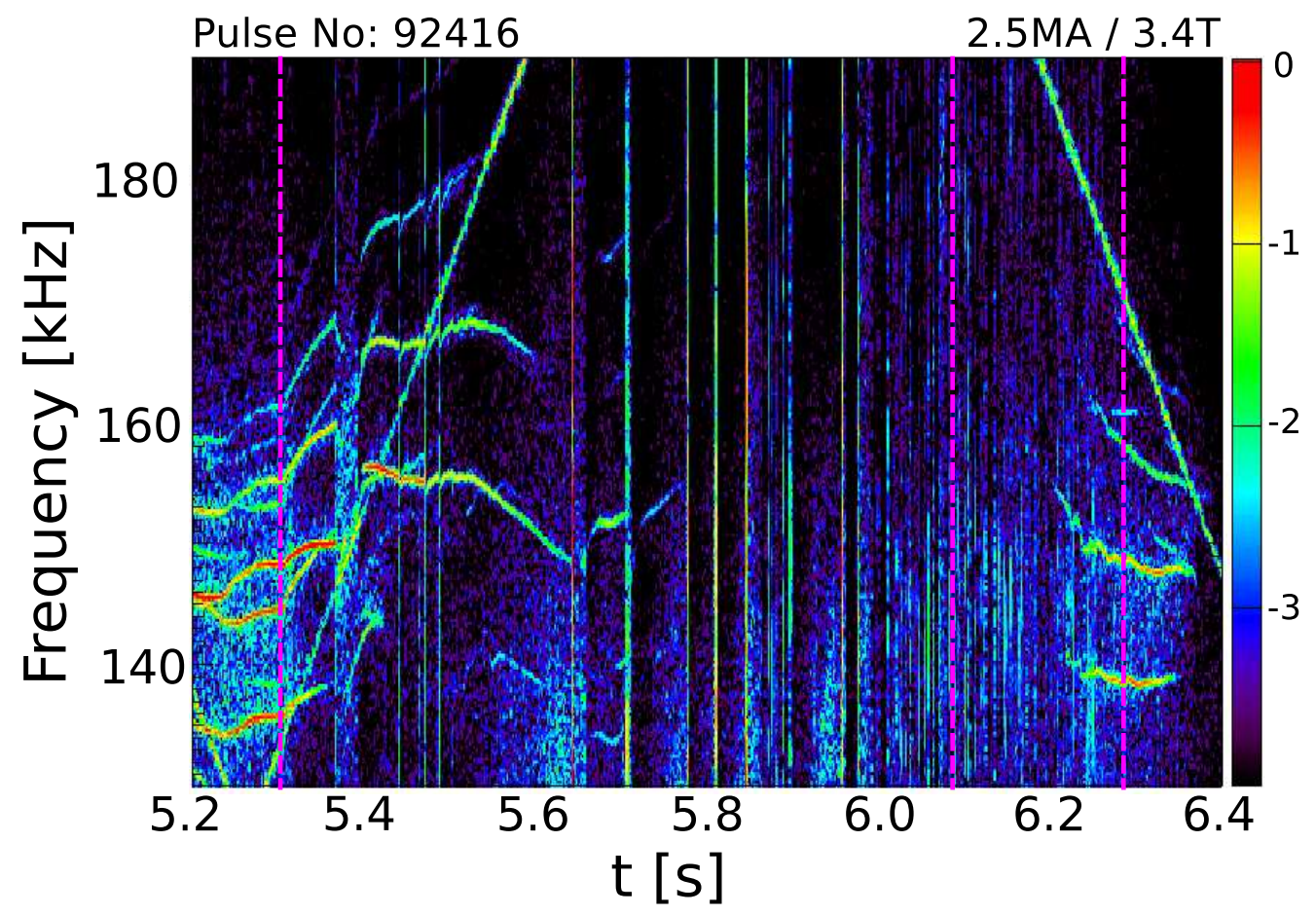

Figure 11. Pulse \#92416. Spectrogram from Mirnov coils. The vertical dashed lines refer to Fig. 10. The oblique lines on the left and right parts of this graph correspond to the TAE antenna signal picked up by the Mirnov coils. 


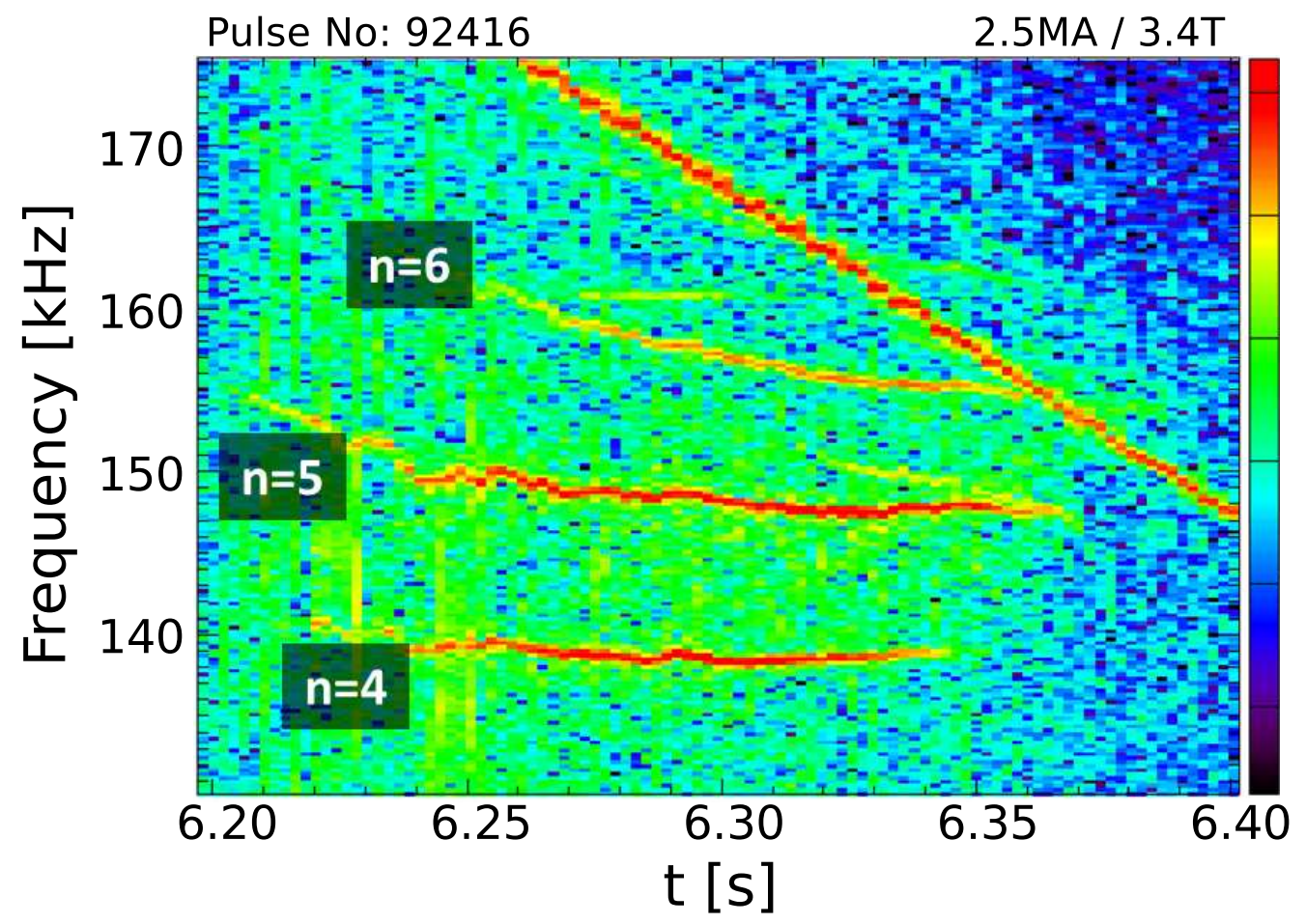

Figure 12. Pulse \#92416. TAEs between time 6.20s and 6.40s corresponding to toroidal mode numbers $n=4,5$ and 6 . The oblique line on the upper right part of this graph is the TAE antenna signal picked up by the Mirnov coils. 
Scenario development for the observation of alpha-driven instabilities in JET DT plasmas27
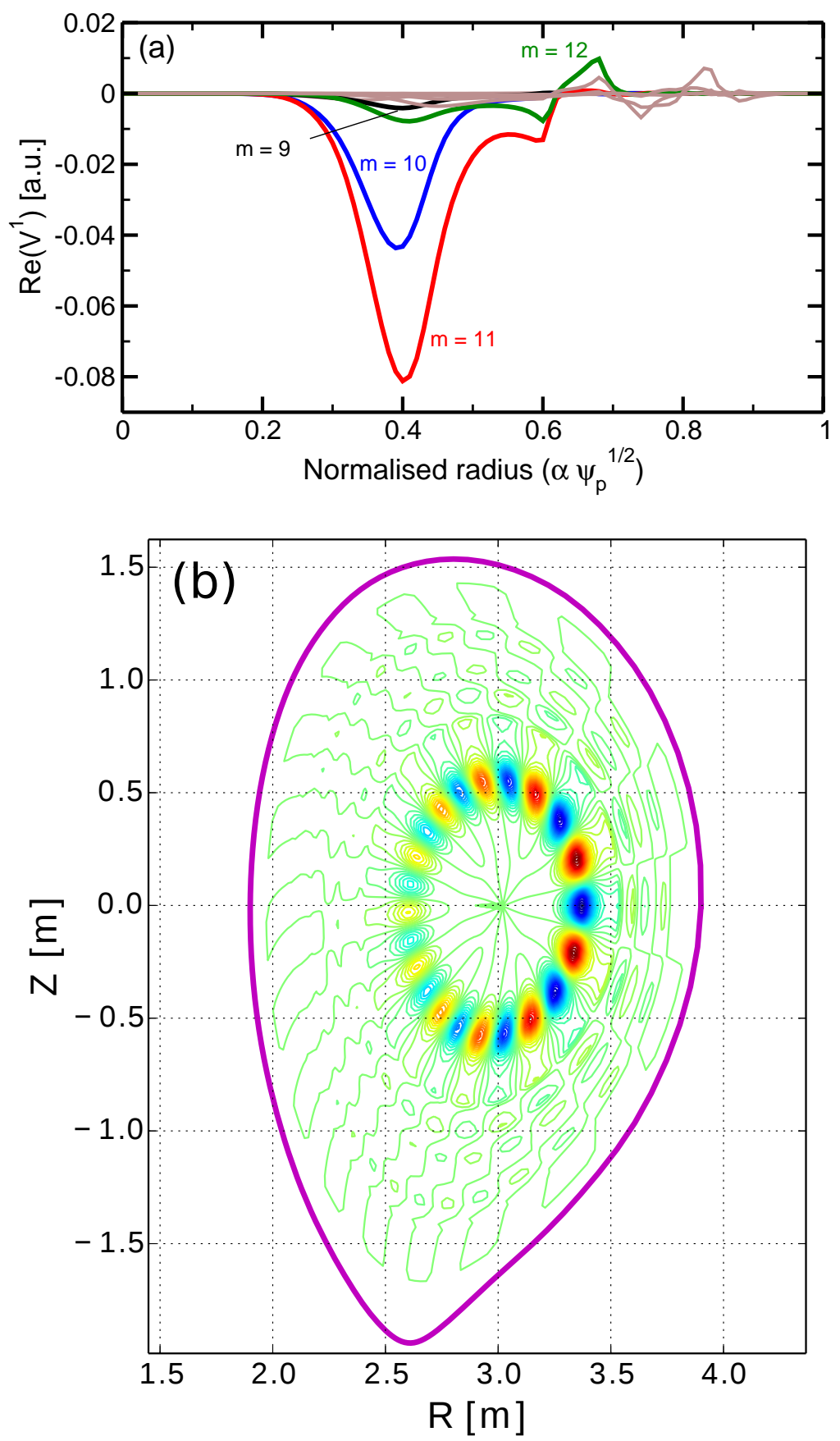

Figure 13. Pulse \#92416, MISHKA calculation corresponding to the $n=5$ TAE shown in Fig. 12 at $t=6.2 s$. (a) Poloidal harmonics of the fluid velocity radial component $V^{1}$; (b) Reconstruction of $V^{1}$ in $(R, Z)$ coordinates. 


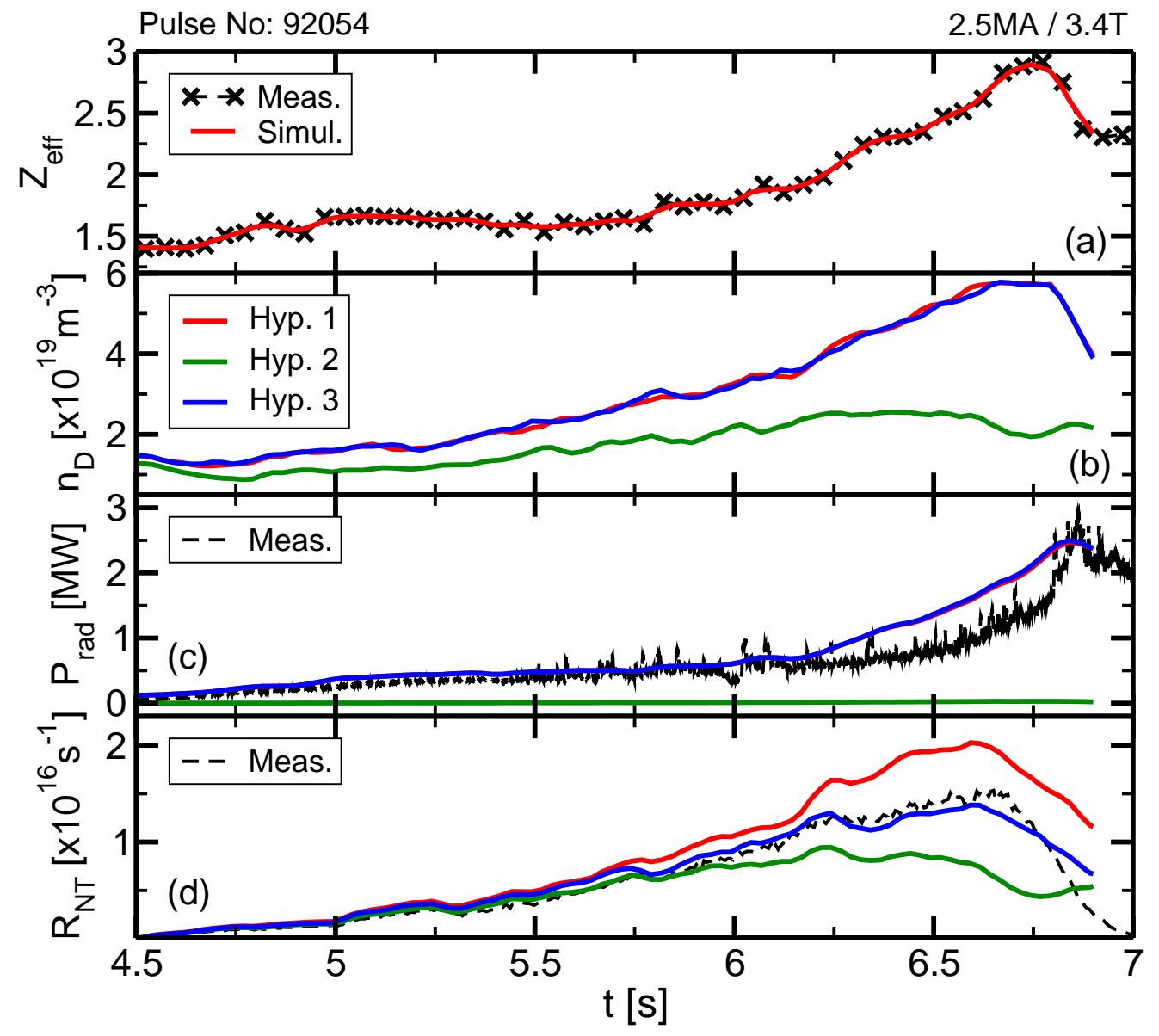

Figure 14. Pulse \#92054 interpretative simulation. (a) Effective charge: measured (symbols), used in TRANSP (solid line); (b) Computed deuterium density; (c) Radiated power: measured (dashed line), computed (solid lines); Neutron rate: measured (dashed line), computed (solid lines). Hyp. 1 (resp Hyp. 2) refers to a plasma with W (resp. B) as the sole impurity; Hyp. 3 refers to a plasma with $\mathrm{W}$ as the sole impurity and a lower ion temperature (see main text). 


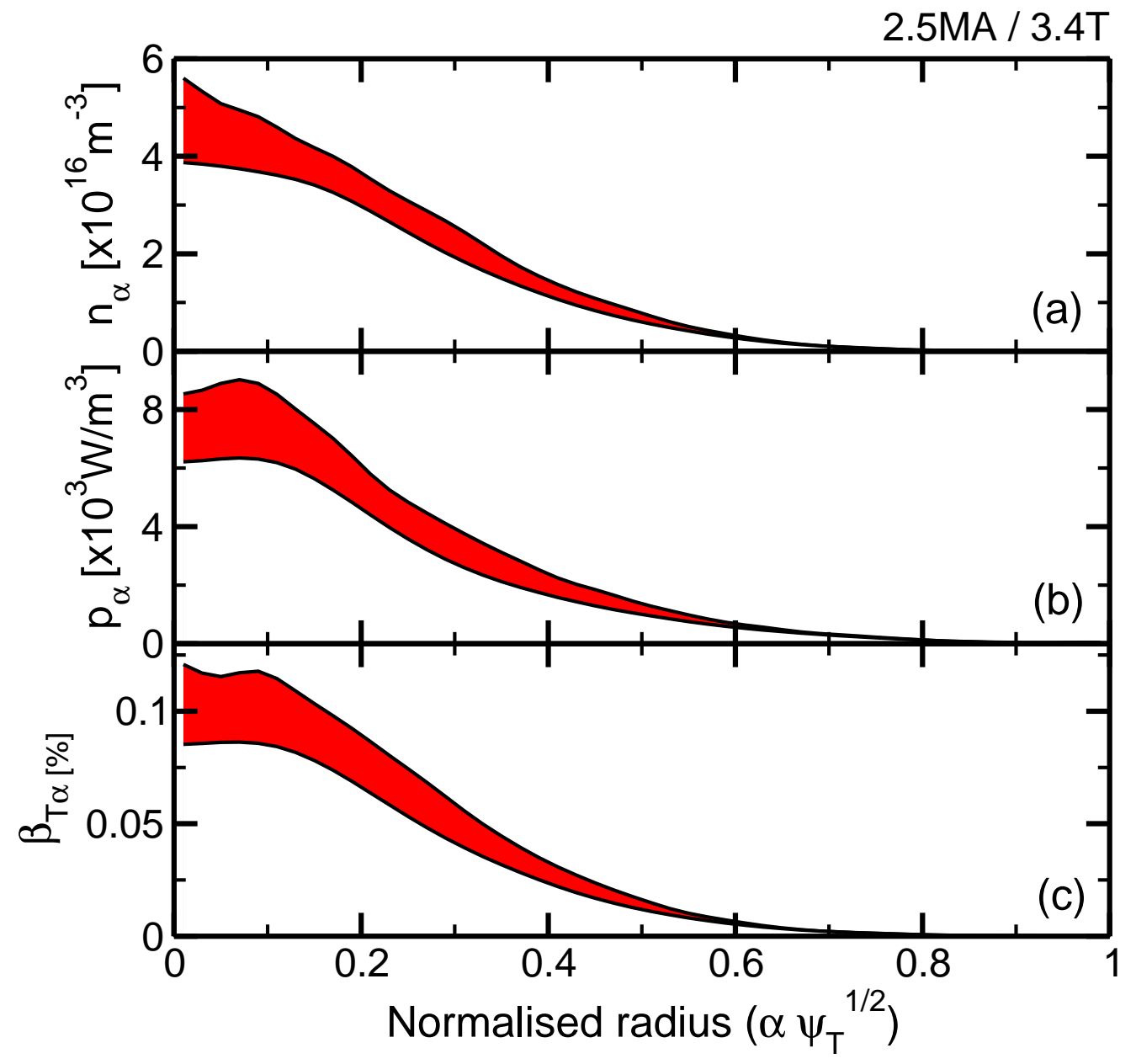

Figure 15. Extrapolation of pulse \#92054 to DT. (a) Fusion alpha density; (b) power source from fusion alphas to bulk plasma particles; (c) normalised fusion alpha beta (toroidal). The shaded area is delimited by hypotheses 1 and 3 in Fig. 14 . 